\title{
Broadening of Inhibitory Tuning Underlies Contrast- Dependent Sharpening of Orientation Selectivity in Mouse Visual Cortex
}

\author{
Ya-tang Li, ${ }^{1,3}$ Wen-pei Ma, ${ }^{1}$ Ling-yun $\mathrm{Li},{ }^{1,4}$ Leena A. Ibrahim, ${ }^{1,4}$ Sheng-zhi Wang, ${ }^{1,4}$ and Huizhong Whit Tao ${ }^{1,2}$ \\ ${ }^{1}$ Zilkha Neurogenetic Institute, ${ }^{2}$ Department of Cell and Neurobiology, ${ }^{3}$ Graduate Program in Physiology and Biophysics, and ${ }^{4}$ Neuroscience Graduate \\ Program, Keck School of Medicine, University of Southern California, Los Angeles, California 90089
}

Orientation selectivity $(\mathrm{OS})$ in the visual cortex has been found to be invariant to increases in stimulus contrast, a finding that cannot be accounted for by the original, purely excitatory Hubel and Wiesel model. This property of OS may be important for preserving the quality of perceived stimulus across a range of stimulus intensity. The synaptic mechanisms that can prevent a broadening of oS caused by contrast-dependent strengthening of excitatory inputs to cortical neurons remain unknown. Using in vivo loose-patch recordings, we found in excitatory neurons in layer 4 of mouse primary visual cortex (V1) that the spike response to the preferred orientation was elevated as contrast increased while that to the orthogonal orientation remained unchanged, resulting in an overall sharpening rather than a weakening of OS. Whole-cell voltage-clamp recordings further revealed that contrast increases resulted in a scaling up of excitatory conductance at all stimulus orientations. Inhibitory conductance was enhanced at a similar level as excitation for the preferred orientation, but at a significantly higher level for the orthogonal orientation. Modeling revealed that the resulting broadening of inhibitory tuning is critical for maintaining and sharpening $O S$ at high contrast. Finally, two-photon imaging guided recordings from parvalbuminpositive (PV) inhibitory neurons revealed that the broadening of inhibition can be attributed to a contrast-dependent broadening of spike-response tuning of PV neurons. Together our results suggest that modulation of synaptic inhibition in the mouse V1 cortical circuit preserves the sharpness of response selectivity during changes of stimulus strength.

\section{Introduction}

Orientation selectivity (OS) in the visual cortex of several mammalian species has been found to be contrast invariant, as demonstrated by a constant tuning bandwidth in the face of increasing stimulus contrast (Movshon et al., 1978; Albrecht and Hamilton, 1982; Sclar and Freeman, 1982; Li and Creutzfeldt, 1984; Skottun et al., 1987; Alitto and Usrey, 2004; Niell and Stryker, 2008). Moreover, recent studies indicate that OS sometimes even exhibits a contrast-dependent sharpening (Alitto and Usrey, 2004; Johnson et al., 2008). These experimental observations contradict the prediction of a broadening of OS at high contrast by a purely feedforward excitatory circuit, caused by an increased drive from nonselective thalamocortical inputs (Hubel and Wiesel, 1962; Troyer et al., 1998; Ferster and Miller, 2000). Two mechanisms have been proposed to explain the contrastdependent properties of OS. First, cortical inhibition among neurons with different orientation tuning or from untuned inhibitory neurons is used in theoretical network models to

Received July 6, 2012; revised Aug. 29, 2012; accepted Sept. 22, 2012.

Author contributions: H.W.T. designed research; Y.-t.L., W.-p.M., L.-y.L., S.-z.W. and L.A.I. performed research; Y.-t.L. and W.-p.M. analyzed data; H.W.T. wrote the paper.

This work was supported by grants to H.W.T. from the United States National Institutes of Health (EY019049 and EY022478) and the Kirchgessner Foundation. We thank Dr. Kurt Zhang for his help on bootstrap analysis.

Correspondence should be addressed to H.W. Tao, USC Keck School of Medicine, 1501 San Pablo Street, ZNI 439, Los Angeles, CA 90033. E-mail: hta0@usc.edu.

DOI:10.1523/JNEUROSCI.3221-12.2012

Copyright $\odot 2012$ the authors $\quad 0270-6474 / 12 / 3216466-12 \$ 15.00 / 0$ achieve contrast invariance (Somers et al., 1995; Troyer et al., 1998; Lauritzen and Miller, 2003). Second, evidence shows that the variability of visually evoked membrane potential responses from trial to trial and the dependence of this variability on contrast can account for contrast invariance (Anderson et al., 2000; Finn et al., 2007; Sadagopan and Ferster, 2012). According to the variability model, pure excitatory mechanisms explain contrast invariance. But this model does not rule out the possible role of inhibition. This is because the membrane potential response results from the integration of visually evoked excitatory and inhibitory conductances, and several intracellular recording studies demonstrate that there can be substantial temporal overlaps between these conductances (Borg-Graham et al., 1998; Liu et al., 2010; Tan et al., 2011; Li et al., 2012). In addition, there has been ample evidence from both extracellular and intracellular studies suggesting that cortical inhibition may play a role in sharpening OS (De Valois et al., 1982; Celebrini et al., 1993; Monier et al., 2003; Ringach et al., 2003; Mariño et al., 2005; Xing et al., 2011). To address whether and how cortical inhibition contributes to maintaining sharp OS in the face of increasing contrast, it is essential to elucidate the inhibitory response tuning at different contrasts, and the responses of inhibitory neurons themselves to different contrasts as a function of orientation. Such studies are still lacking.

Thanks to the availability of recording techniques to dissect synaptic inputs and of genetic tools to target inhibitory neurons, the mouse has now become an important model to understand 
the mechanisms for visual processing (Taniguchi et al., 2011). Mouse visual cortical neurons can be as sharply tuned for orientation as cat cells (Niell and Stryker, 2008; Liu et al., 2009; Tan et al., 2011). Our previous study of mouse simple cells at a relatively high contrast indicates that their OS is significantly sharpened by very broadly tuned inhibition (Liu et al., 2011). Here we report that excitatory and parvalbumin-positive (PV) inhibitory neurons undergo contrast-dependent sharpening and broadening of OS respectively, and that a broadening of inhibitory synaptic tuning contributes critically to maintaining sharp OS at high contrast.

\section{Materials and Methods}

Animal preparation. All experimental procedures used in this study were approved by the Animal Care and Use Committee of the University of Southern California. Adult female wild-type C57BL/6 mice (2-3 months) were used for voltage-clamp recordings and some loose-patch recordings. For visually guided recordings from layer 4, the layer 4-specific Cre mouse line, Scnn1a-Tg3-Cre (The Jackson Laboratory), was crossed with the Ai9 reporter line, which contains the DIOtdTomato at ROSA26 locus (The Jackson Laboratory). For recordings from PV neurons, the mice with PV neurons expressing tdTomato were obtained by crossing the PV-ires-Cre driver line (The Jackson Laboratory) with the Ai9 reporter line. Mice were sedated with an intraperitoneal injection of chlorprothixene $(5 \mathrm{mg} / \mathrm{kg})$ and then anesthetized with urethane $(0.5 \mathrm{~g} / \mathrm{kg}$, i.p., at $10 \% \mathrm{w} / \mathrm{v}$ in saline), as previously described (Niell and Stryker, 2008; Liu et al., 2009). The animal's body temperature was maintained at $\sim 37.5^{\circ} \mathrm{C}$ by a heating pad (Harvard Apparatus). A tracheotomy was performed, and a small glass capillary tube was inserted to maintain a free airway. CSF draining was performed. The part of the skull and dura mater $(\sim 1 \times 1 \mathrm{~mm})$ over the primary visual cortex $(\mathrm{V} 1)$ was removed. An artificial CSF solution [ACSF, containing (in mM): 140 $\mathrm{NaCl}, 2.5 \mathrm{KCl}, 2.5 \mathrm{CaCl}_{2}, 1.3 \mathrm{MgSO}_{4}, 1.0 \mathrm{NaH}_{2} \mathrm{PO}_{4}, 20$ HEPES, 11 glucose, $\mathrm{pH}$ 7.4] was applied to the exposed cortical surface when necessary. The eyes were covered with ophthalmic lubricant ointment until recording, at which time the eyes were rinsed with saline and a thin layer of silicone oil (30,000 centistokes) was applied to prevent drying while allowing clear optical transmission. Eye movements and the receptive field drift of single units were negligible within the time window of our recordings (Mangini and Pearlman, 1980; Liu et al., 2010).

In vivo electrophysiology. The pia was prepenetrated with a broken pipette under visual guidance before recording experiments. Whole-cell voltage-clamp recordings were performed with an Axopatch 200B (Molecular Devices). The patch pipette had a tip opening of $\sim 2 \mu \mathrm{m}$ (4.5-6 $\mathrm{M} \Omega$ impedance). The $\mathrm{Cs}^{+}$-based intrapipette solution used for voltageclamp recordings contained the following (in $\mathrm{mM}$ ): $125 \mathrm{Cs}$-gluconate, 5 TEA-Cl, 4 MgATP, 0.3 GTP, 8 phosphocreatine, 10 HEPES, 10 EGTA, 2 CsCl, 1 QX-314, 0.75 MK-801, pH 7.25. The $\mathrm{K}^{+}$-based intrapipette solution used for current-clamp recordings contained the following (in $\mathrm{mm}$ ): $130 \mathrm{~K}$-gluconate, $2 \mathrm{KCl}, 1 \mathrm{CaCl}_{2}, 4 \mathrm{MgATP}, 0.3 \mathrm{GTP}, 8$ phosphocreatine, 10 HEPES, 11 EGTA, pH 7.25. The pipette capacitance and whole-cell capacitance were compensated completely, and series resistance (25-50 M $\Omega$ ) was compensated by $50-60 \%$ (100 $\mu \mathrm{s} \mathrm{lag).} \mathrm{A} 12 \mathrm{mV}$ junction potential was corrected. Signals were filtered at $2 \mathrm{kHz}$ for the voltage-clamp recording and $5 \mathrm{kHz}$ for the current-clamp recording, and sampled at $10 \mathrm{kHz}$. The evoked excitatory and inhibitory currents were resolved by clamping the cell at -70 and $0 \mathrm{mV}$, respectively. As discussed previously (Liu et al., 2010), our whole-cell recording method highly biases sampling toward pyramidal neurons. For cell-attached recordings, glass electrodes containing ACSF were used. A 100-250 M $\Omega$ seal was formed on the targeted neuron. The pipette capacitance was completely compensated. Spikes were recorded in the voltage-clamp mode, with a command potential applied to achieve a zero baseline current. The spike signal was filtered at $10 \mathrm{kHz}$ and sampled at $20 \mathrm{kHz}$. The blindly recorded neurons assigned to layer 4 in this study had a cortical depth ranging from 375 to $520 \mu \mathrm{m}$, which was predicted from the travel distance of the pipette in the tissue. The layer assignment was reasonably precise for three reasons. First, the quantification of the layer 4 location using visual cortical slices of the layer 4-specific reporter mice, Scnn1a-Tg3-Cre td-
Tomato, demonstrated that the upper and lower boundaries of layer 4 were at $374 \pm 14 \mu \mathrm{m}$ and $510 \pm 16 \mu \mathrm{m}$ (mean \pm SD, $n=3$ brains, 15 locations) beneath the pial surface, respectively. This was confirmed by Nissl staining (data not shown). Second, the use of a high-magnification objective $(40 \times)$ on our custom-built microscope enabled a precise identification of the cortical surface. In addition, the application of prepenetration greatly reduced the dimpling of the cortical surface in our experiments. Third, under two-photon imaging and with the use of a dye-filled pipette, we quantified the deviation of the predicted depth from the actual depth. At a depth of 350-465 $\mu \mathrm{m}$, there was a small systematic error of predicted depths $(14 \pm 5 \mu \mathrm{m}$, mean $\pm \mathrm{SD}, n=10$ brains). This systematic error was applied to correct the predicted depth. The identified excitatory neurons had a trough-to-peak interval of $0.89 \pm 0.09 \mathrm{~ms}$ (mean $\pm \mathrm{SD}$, see Fig. $1 A$, inset) in their spike waveforms.

In vivo two-photon imaging guided recording. Two-photon imaging was performed with a custom-built imaging system as previously described (Liu et al., 2009). A mode-locked Ti:sapphire laser (MaiTai Broadband, Spectra-Physics) was tuned at $890 \mathrm{~nm}$ with the output power at $60-300 \mathrm{~mW}$ for imaging fluorescently labeled neurons in layer 4 , adjusted according to the cell's fluorescence level. The glass electrode, with $\sim 1 \mu \mathrm{m}$ tip opening and $8-10 \mathrm{M} \Omega$ impedance, was filled with ACSF containing $0.15 \mathrm{~mm}$ calcein (Invitrogen). The pipette capacitance was completely compensated. The pipette tip was navigated in the cortex and patched onto a fluorescent soma as previously described (Liu et al., 2009). After confirming a successful targeting, the positive pressure in the pipette ( $\sim 10 \mathrm{mbar})$ was then released and a negative pressure $(20-150$ mbar) was applied to form a loose seal (with 80-200 $\mathrm{M} \Omega$ resistance), which was maintained throughout the course of the recording. The depth of the patched cell could be directly determined under the two-photon microscope. The depth of the recorded PV neurons in this study ranged from 350 to $465 \mu \mathrm{m}$ beneath the pia. The recorded PV neurons all exhibited narrow spike waveforms, with an average through-to-peak interval of $0.30 \pm 0.04 \mathrm{~ms}$.

Visual stimulation. Stimuli were created using Matlab with Psychophysics Toolbox and displayed with a gamma-corrected LCD monitor (refresh rate, $75 \mathrm{~Hz}$; maximum luminance, $280 \mathrm{~cd} / \mathrm{m}^{2}$ ) placed $0.25 \mathrm{~m}$ away from the right eye. The center of the monitor was placed at $45^{\circ}$ azimuth, $0^{\circ}$ elevation, and it covered $\pm 35^{\circ}$ horizontally and $\pm 27^{\circ}$ vertically of the mouse visual field. Recordings were mostly made in the monocular zone of the V1. Spontaneous activity was recorded when a uniform gray background was applied. To measure orientation tuning, drifting sinusoidal gratings $\left(2 \mathrm{~Hz}, 0.04\right.$ cycle/degree) of 12 directions $\left(30^{\circ}\right.$ step) and different contrasts were applied in a random sequence. A stationary grating of one orientation was first presented on the full screen for $1.8 \mathrm{~s}$ before it drifted for $1.5 \mathrm{~s}$ ( 3 cycles). The grating stopped drifting for $500 \mathrm{~ms}$ before another grating pattern appeared. The 12 patterns were presented in a random sequence. Ten sets of stimuli were applied to each cell, with the sequence different between sets. The maximum contrast for the drifting gratings achieved by the monitor was 95\% (near 100\%).

Data analysis. The data acquisition software was custom-developed with LabVIEW (National Instrument). Spikes evoked by drifting gratings were counted within a $70-1570 \mathrm{~ms}$ window after the start of drifting. The spontaneous firing rate was subtracted from the stimulus-evoked spike rate. The recorded synaptic responses were cycle-averaged, and then were smoothed by averaging within a $40 \mathrm{~ms}$ sliding window. The response levels for drifting sinusoidal gratings of two directions at the same orientation were averaged to obtain the orientation tuning curve between 0 and $180^{\circ}$, which was then fit with a Gaussian function $R(\theta)=A^{*}$ $\exp \left(-0.5^{*}(\theta-\varphi)^{2} / \sigma^{2}\right)+B$, with $\varphi$ the preferred orientation and $\sigma$ the tuning width. The strength of orientation selectivity was quantified with a global measure of orientation selectivity index:

\section{Global OSI}

$$
=\sqrt{\left(\sum_{i}\left(R\left(\theta_{i}\right) * \sin \left(2 \theta_{i}\right)\right)\right)^{2}+\left(\sum_{i}\left(R\left(\theta_{i}\right) * \cos \left(2 \theta_{i}\right)\right)\right)^{2}} / \sum_{i} R\left(\theta_{i}\right)
$$

where $\theta_{\mathrm{i}}$ is the angle of the moving direction of the grating and $R\left(\theta_{\mathrm{i}}\right)$ is the response level at angle $\theta_{\mathrm{i}}$. 


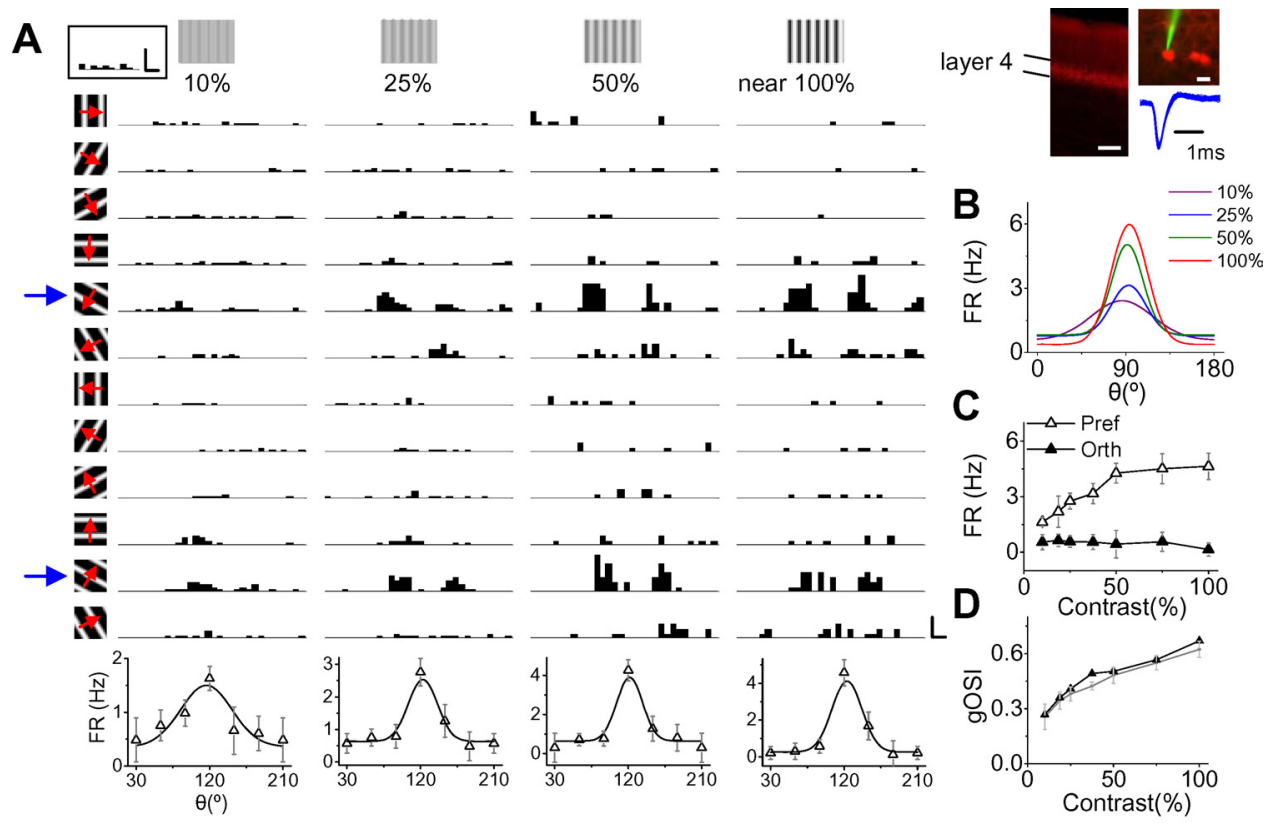

E
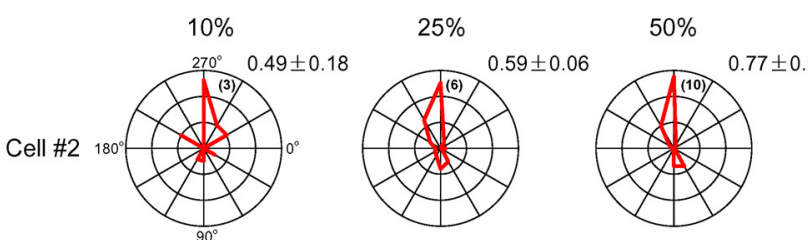

near $100 \%$
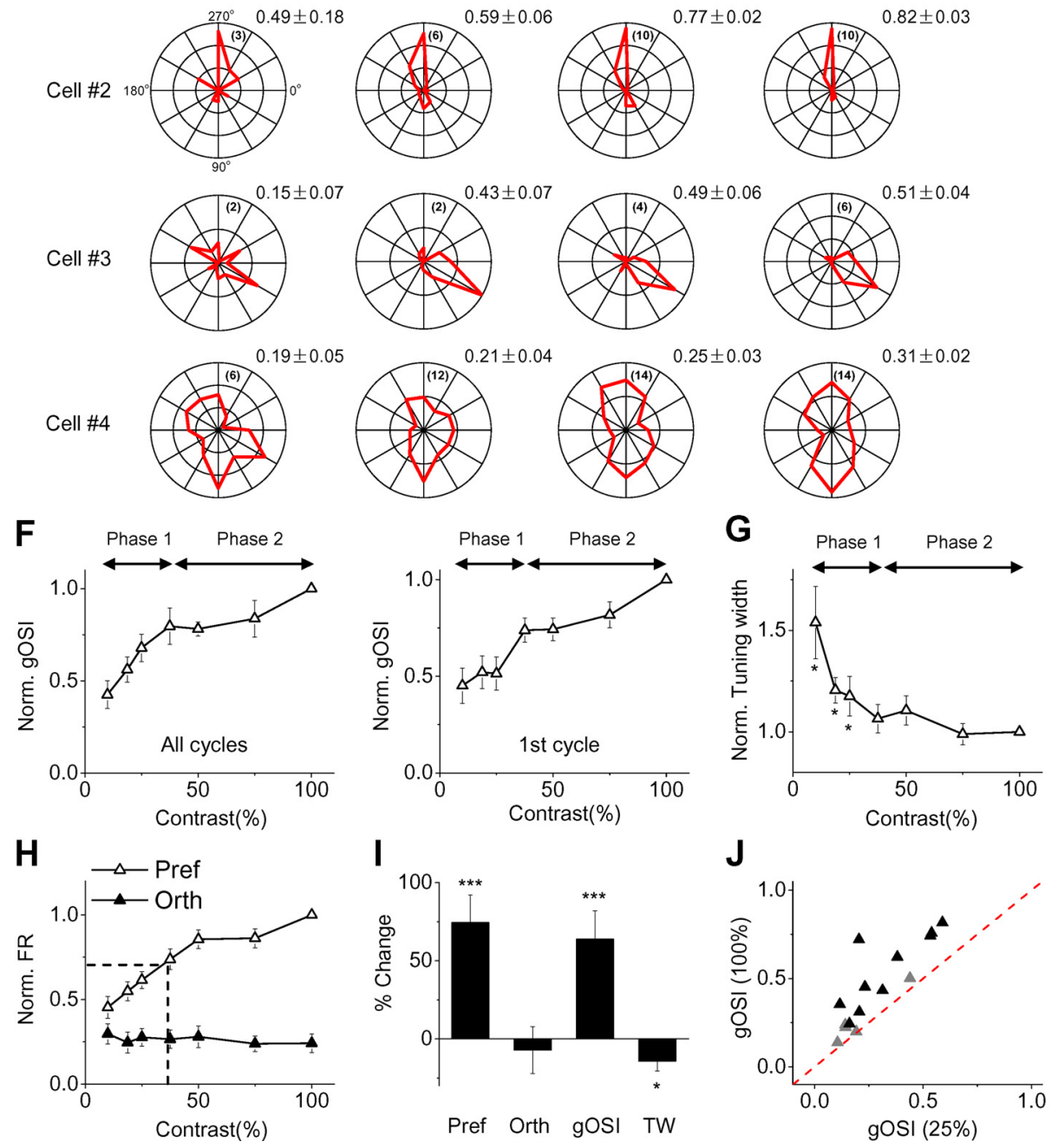

Figure 1. Contrast-dependent changes of orientation selectivity of layer 4 excitatory neurons in mouse visual cortex. A, Spike responses of an example layer 4 neuron to drifting sinusoidal gratings of different contrasts (labeled on the top), recorded in a Scnn1a-Tg3-Cre tdTomato mouse. Each trace (1.5 s, 3 cycles) represents the poststimulus spike time histograms (PSTHs) from 10 trials for a particular stimulus. Calibration: $30 \mathrm{~Hz}, 100 \mathrm{~ms}$. Blue arrows mark the preferred orientation. Bottom, Tuning curves of average spike rate within the three cycles. Bar, SD. Gaussian fits of the tuning curves are shown. Boxed inset, Top left, PSTH for spontaneous activity. Calibration: $3 \mathrm{~Hz}, 100 \mathrm{~ms}$. Inset, Top right, Coronal section of the cortex showing specific labeling of neurons in layer 4 (left; scale bar, $200 \mu \mathrm{m}$ ), a two-photon image of the patched layer 4 cell (upper right; scale bar, $20 \mu \mathrm{m}$ ), and superimposed 50 individual spike waveforms of the cell (Figure legend continues.) 
In voltage-clamp recordings, excitatory and inhibitory synaptic conductances were derived according to the following equation (Zhang et al., 2003; Tan et al., 2004; Liu et al., 2007; Wu et al., 2008): $\Delta I(t)=G_{e}(t)\left(V(t)-E_{e}\right)+G_{i}(t)\left(V(t)-E_{i}\right)$, where $\Delta I(t)$ is the amplitude of synaptic current at a time point, $G_{e}$ and $G_{i}$ are the excitatory and inhibitory synaptic conductance respectively, $V(t)$ is the membrane voltage, and $E_{\mathrm{e}}(0 \mathrm{mV})$ and $E_{\mathrm{i}}(-70 \mathrm{mV})$ are the reversal potentials. $V(t)$ is corrected by $V(t)=V_{\mathrm{h}}-R_{\mathrm{s}}{ }^{*} I(t)$, where $R_{\mathrm{s}}$ was the effective series resistance and $V_{\mathrm{h}}$ is the applied holding voltage.

The estimated membrane potential response based on the synaptic conductances was derived using a single-compartment neuron model (Somers et al., 1995; Troyer et al., 1998; Liu et al., 2010; Zhang et al., 2011):

$$
\begin{aligned}
V_{m}(t+d t)=-\frac{d t}{C}\left[G_{e}(t) *\left(V_{m}(t)-E_{e}\right)+\right. & G_{i}(t) *\left(V_{m}(t)-E_{i}\right) \\
& \left.+G_{r}\left(V_{m}(t)-E_{r}\right)\right]+V_{m}(t)
\end{aligned}
$$

where $V_{\mathrm{m}}(t)$ is the membrane potential at time $t, C$ the whole-cell capacitance, $G_{\mathrm{r}}$ the resting leak conductance, and $E_{\mathrm{r}}$ the resting membrane potential. $C$ was measured during the experiment. $G_{\mathrm{r}}$ and $E_{\mathrm{r}}$ were calculated based on the baseline currents at two potentials.

Modeling. The synaptic conductance evoked by a moving grating was simulated by fitting the average synaptic response waveform with a skewnormal function (Li et al., 2012), which yielded a better fit than sinusoidal functions or $\alpha$ functions (data not shown):

$$
f(x)=\text { amplitude } \times \frac{2}{\omega} \phi\left(\frac{x-\xi}{\omega}\right) \times \Phi\left(\alpha\left(\frac{x-\xi}{\omega}\right)\right)+\text { baseline }
$$

where $\phi$ and $\Phi$ are the standard normal probability density function and the cumulative distribution function respectively. $\xi$ determines the location. The scale factor $(\omega)$ was set at $145 \mathrm{~ms}$ and the shape factor $(\alpha)$ at 1.5 for both excitatory and inhibitory conductances. To generate a relatively fast rising phase of the conductance change, the synaptic conductance was set as 0 if it was smaller than $10 \%$ of maximum. Because the phase difference between excitation and inhibition was $21 \pm 15^{\circ}$ (mean \pm SD, $n=14$ ), inhibition had a $25 \mathrm{~ms}$ delay relative to that of excitation (corresponding to a $18^{\circ}$ phase difference). Varying the relative delay of inhibition from 5 to $50 \mathrm{~ms}$ did not qualitatively affect our conclusion (data not shown). To simulate responses to different orientations, while maintaining the temporal shape of the evoked conductance, the peak amplitude of the evoked conductance was varied according to the simulated tuning curve created based on the average synaptic tuning curve in our experimental data. The amplitudes of optimally evoked excitatory and inhibitory conductances were set based on the average experimental data $(1.37 \mathrm{nS}$ for excitation and $2.94 \mathrm{nS}$ for inhibition at $25 \%$ contrast; $2.14 \mathrm{nS}$ and $4.43 \mathrm{nS}$ at near $100 \%$ contrast). The same conductance-based neuron model described earlier was used to simulate the membrane potential

\section{$\leftarrow$}

(Figure legend continued.) (lower right). Note that the interval between the trough and the peak is relatively long ( $>0.5 \mathrm{~ms})$. B, Superimposed fitted tuning curves at different contrasts. $\boldsymbol{C}$, The average spike rates at the preferred and orthogonal orientations plotted against contrast for the same cell. $\boldsymbol{D}, \mathrm{g}$ gSI plotted against contrast for the same cell. Black symbols, gOSI measured from average observed values. Gray symbols, Mean and SD of bootstrapped gOSIs (resamplings, 1000 times). $\boldsymbol{E}$, Polar graph of evoked spike rate for another three example cells. The maximum axis value is labeled in the parentheses. The mean $\pm S D$ of bootstrapped goSIs is shown for each cell. $\boldsymbol{F}$, Average gOSI (normalized to the value at near $100 \%$ contrast) plotted against contrast. Left, gOSI based on spike rate within all cycles. Right, gOSI based on spike rate within the first cycle. Bar, SE; $n=15$ cells. G, Average tuning width (normalized to value at near $100 \%$ contrast) plotted against contrast. ${ }^{*} p<0.05$, paired $t$ test, compared with near $100 \%$ contrast. $\boldsymbol{H}$, Average evoked spike rate (normalized to the value of the preferred orientation at near $100 \%$ contrast) at the preferred and orthogonal orientations. Dash line, Contrast at which the increase in spike rate at the preferred orientation has reached half maximum. $I$, Percentage change in evoked spike rate (preferred and orthogonal orientation), gOSI, and tuning width from $25 \%$ to near $100 \%$ contrast. ${ }^{*} p<0.05 ;{ }^{* * *} p<0.001$, paired $t$ test.J, Plot of the mean of bootstrapped gOSIs at near $100 \%$ contrast versus that at $25 \%$ contrast for individual cells. Solid black, Cells that exhibit significant deviation from the identity line (bootstrap test, $p<0.01$ ). response generated by the integration of model synaptic conductances. $E_{\mathrm{r}}$ was $-65 \mathrm{mV}$. $E_{\mathrm{e}}$ and $E_{\mathrm{i}}$ were $0 \mathrm{mV}$ and $-75 \mathrm{mV}$, respectively. $C$ was 20 pF. $G_{\mathrm{r}}$ was calculated based on the equation $G_{\mathrm{r}}=C^{\star} G_{\mathrm{m}} / C_{\mathrm{m}}$, where $G_{\mathrm{m}}$, the specific membrane conductance, is $2 \mathrm{e}-5 \mathrm{~S} / \mathrm{cm}^{2}$, and $C_{\mathrm{m}}$, the specific membrane capacitance, is $1 \mathrm{e}-6 \mathrm{~F} / \mathrm{cm}^{2}$.

\section{Results}

\section{Contrast-dependent sharpening of OS of excitatory neurons}

To first understand contrast-dependent orientation tuning properties of mouse visual cortical neurons, we performed in vivo loose-patch recordings in the V1. Since contrast invariance is a prominent problem for layer 4 neurons receiving direct thalamic inputs (Ferster and Miller, 2000), we focused our study in layer 4. Taking advantage of a layer 4-specific Cre driver line, Scnn1aTg3-Cre (Madisen et al., 2010), we performed visually guided recordings from layer 4 neurons labeled by expressing a red fluorescence protein, tdTomato, under a two-photon microscope (see Materials and Methods). Spike responses of the patched neuron to drifting sinusoidal gratings of different contrasts ranging from $10 \%$ to near $100 \%$ were recorded, as shown by an example cell (Fig. 1A). The broad spike waveforms (Fig. 1A, inset) indicated that it was most likely an excitatory neuron (Liu et al., 2009). With contrast increasing, the spike response of the cell (as measured by the average spike rate within all stimulus cycles) to the preferred orientation increased drastically, while that to the orthogonal orientation did not (Fig. $1 A, B$ ). The tuning curve of evoked spike rate appeared sharper at $25 \%$ than at $10 \%$ contrast, as evidenced by the narrower tuning width. Higher than $25 \%$ contrast, the tuning width changed little. The plot of evoked spike rate at all testing contrasts shows that spike rate at the preferred orientation increased with increasing contrast, while that at the orthogonal orientation barely changed (Fig. 1C). The sharpness of tuning, as quantified with a global orientation selectivity index (gOSI, see Materials and Methods), was steadily increased from low to high contrast (Fig. 1D). Figure $1 E$ shows the polar graph plots of responses of three example excitatory neurons recorded blindly in wild-type mice (see Materials and Methods). In general, they showed similar contrastdependency as the cell in Figure $1 A$. The first two cells were sharply tuned. Their tuning sharpness was apparently stable between $25 \%$ and near $100 \%$ contrast. The third cell was only moderately tuned and had a higher response level compared with the first two. Nevertheless, its tuning shape was not dramatically changed by increasing contrast.

Data on layer 4 excitatory neurons either visually recorded in Scnn1a-Tg3-Cre mice $(n=5)$ or blindly recorded in wild-type mice $(n=10)$ were summarized (Fig. $1 F, G)$. There appeared to be two phases for the change of gOSI: an initial phase in which gOSI increased quickly, and a later phase $(>37.5 \%$ contrast $)$ in which gOSI increased more slowly (Fig. $1 F$, left). The average tuning width was decreasing in the initial phase, whereas it was relatively stable in the second phase (Fig. $1 G$ ). Thus, overall, there is a sharpening of orientation tuning with increasing contrast. However, within a range of midcontrast to high contrast $(37.5 \%$ to near $100 \%$ ), the results are consistent with the notion of contrast invariance (i.e., tuning width being constant). There is a drastic difference in contrast-dependency between responses to the preferred and orthogonal orientations: the spike rate at the preferred orientation was elevated with increasing contrast with the half elevation point occurring at $\sim 30 \%$ contrast, whereas that at the orthogonal orientation was on average unchanged (Fig. $1 H)$. Based on these results on spiking response, we chose to compare excitatory and inhibitory synaptic responses between 
A

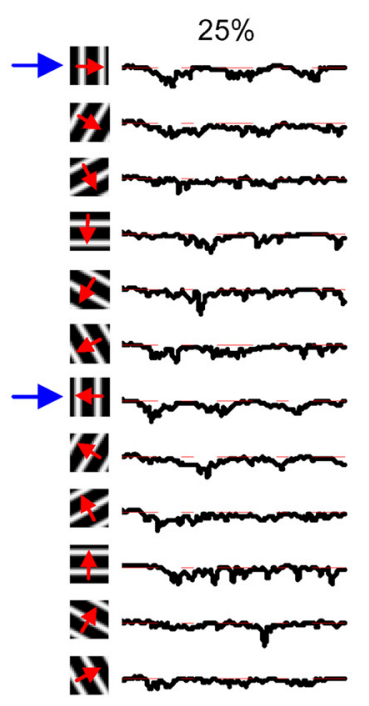

Excitatory (-70mv)

E

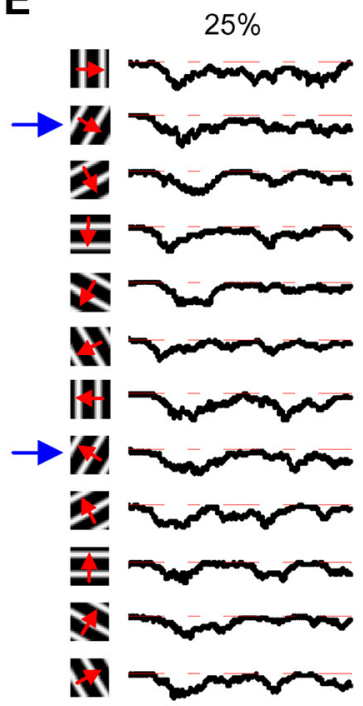

near $100 \%$
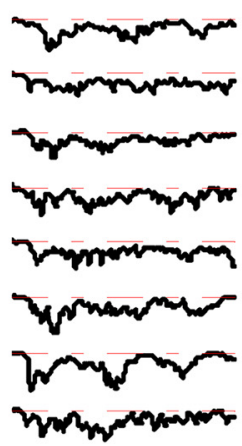

Trempringaminom

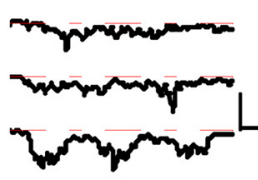

B
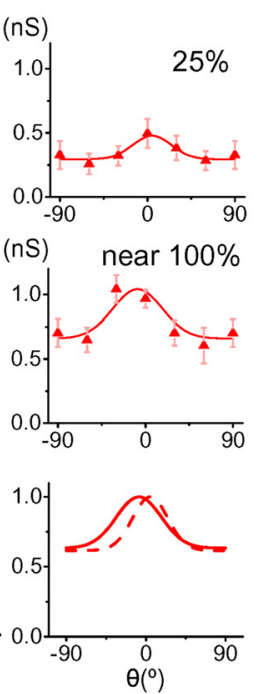

F

near $100 \%$
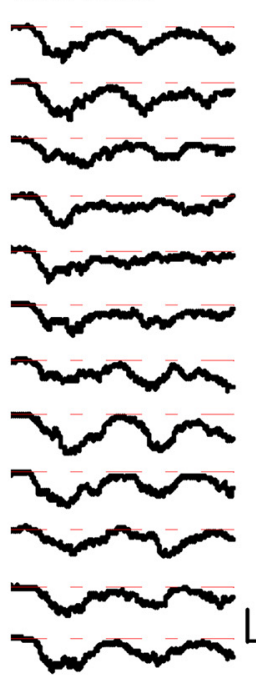

(nS)

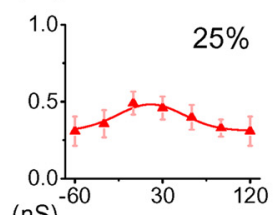

$\begin{array}{lcc}(\mathrm{nS})^{-60} & 30 & 120 \\ & & \end{array}$
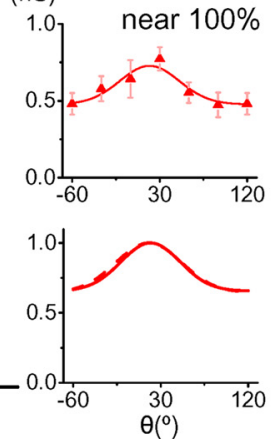

C Inhibitory (0mV)

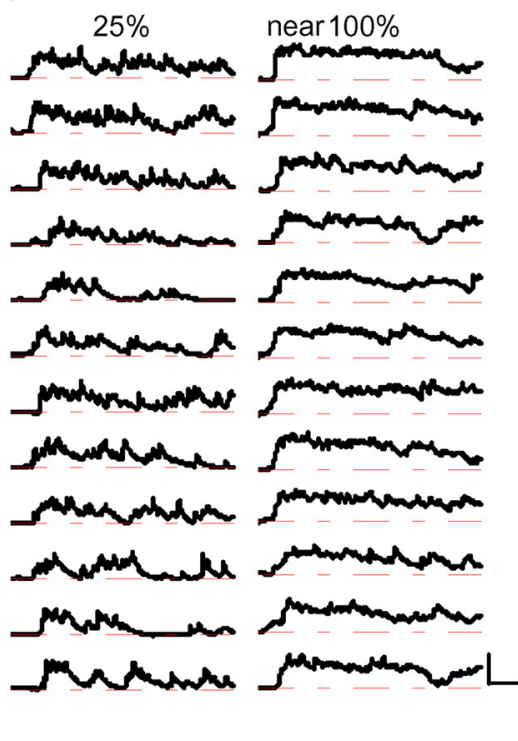

G

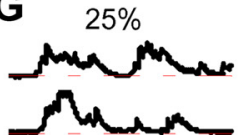

near $100 \%$
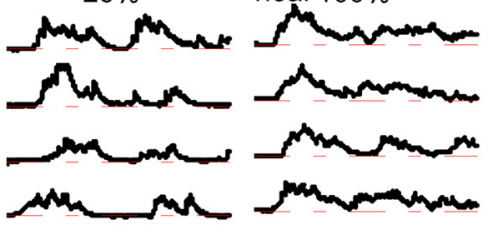

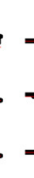
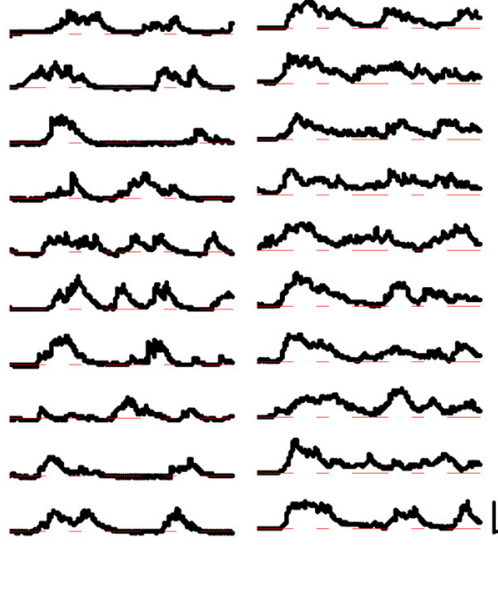

H

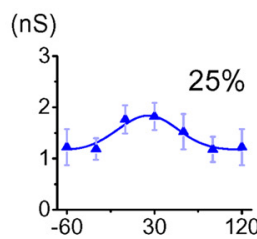

(nS) near $100 \%$
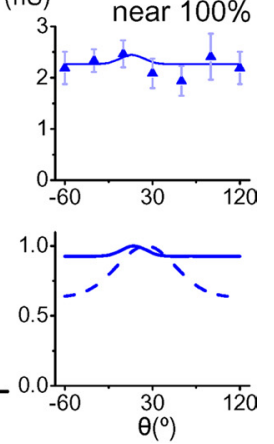

Figure 2. Excitatory and inhibitory synaptic responses revealed by voltage-clamp recordings. A, Average traces of excitatory responses of an example cell at $25 \%$ and near $100 \%$ contrast. Red dash lines, Pretrigger baseline. $\boldsymbol{B}$, Response tuning curve at 25\% (top) and near 100\% (middle) contrast for the same cell. Bottom, Normalized response tuning curve at 25\% (dash) and near 100\% (solid) contrast. $\boldsymbol{C}, \boldsymbol{D}$, Inhibitory responses and tuning curves for the same cell. $\boldsymbol{E}-\boldsymbol{H}$, Synaptic responses and tuning curves for another example cell. Data are presented in a similar manner. Calibrations: $\boldsymbol{A}$, $100 \mathrm{pA} ; C, 200 \mathrm{pA} ; E, 50 \mathrm{pA} ; \mathbf{G}, 150 \mathrm{pA} ; 200 \mathrm{~ms}$.

contrast levels of $25 \%$ and near $100 \%$ in the later experiments. As summarized in Figure 1 , from $25 \%$ to near $100 \%$ contrast, the spike rate at the preferred orientation increased by $74 \pm 18 \%$ (mean $\pm \mathrm{SD}, p<0.001$, paired $t$ test), while that at the orthogonal orientation did not change significantly $(-7 \pm 15 \%, p>0.1$, paired $t$ test). This led to a $63 \pm 18 \%$ increase in the value of gOSI $(p<0.001$, paired $t$ test). In the meantime, tuning width was slightly but significantly reduced ( $p<0.05$, paired $t$ test). In the mouse visual cortex, a reduction in response over time (i.e., adaptation) was often observed (Tan et al., 2011; Li et al., 2012). To avoid potential confounds of changes of OS over time, we also analyzed the spike rate within the first cycle. As shown in Figure $1 F$ (right), the analysis of the first cycle also revealed an increase in gOSI with increasing contrast. Furthermore, we performed bootstrap resamplings (1000 times) of evoked responses so as to overcome some random variation during one stimulus presentation
(Efron and Tibshirani, 1993; Carandini et al., 1997). Figure $1 J$ shows the mean of bootstrapped gOSIs at 25\% and near $100 \%$ contrasts for individual cells. Sixty-seven percent of the cells exhibited a significant increase in gOSI at the high contrast (bootstrap, $p<0.01$ ), while no cell exhibited a decrease in gOSI at the high contrast (Fig. $1 J$ ).

Contrast-dependent excitatory and inhibitory synaptic responses

To dissect visually evoked excitatory and inhibitory synaptic inputs, we performed whole-cell voltage-clamp recordings using a cesiumbased internal solution (see Materials and Methods). As shown by two example cells (Fig. 2A, C,E, G), we recorded excitatory and inhibitory responses to gratings while clamping the cell's membrane potential at two different levels, -70 and $0 \mathrm{mV}$, respectively (Liu et al., 2010,2011). To quantify the strength of synaptic responses, we 

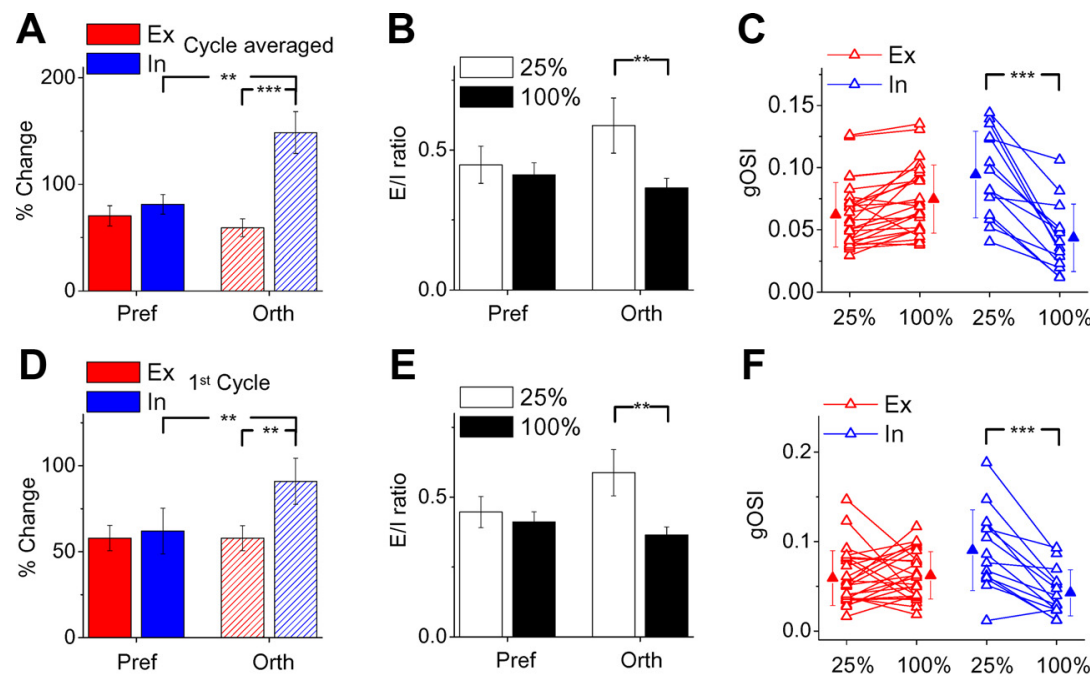

$\mathbf{F}$
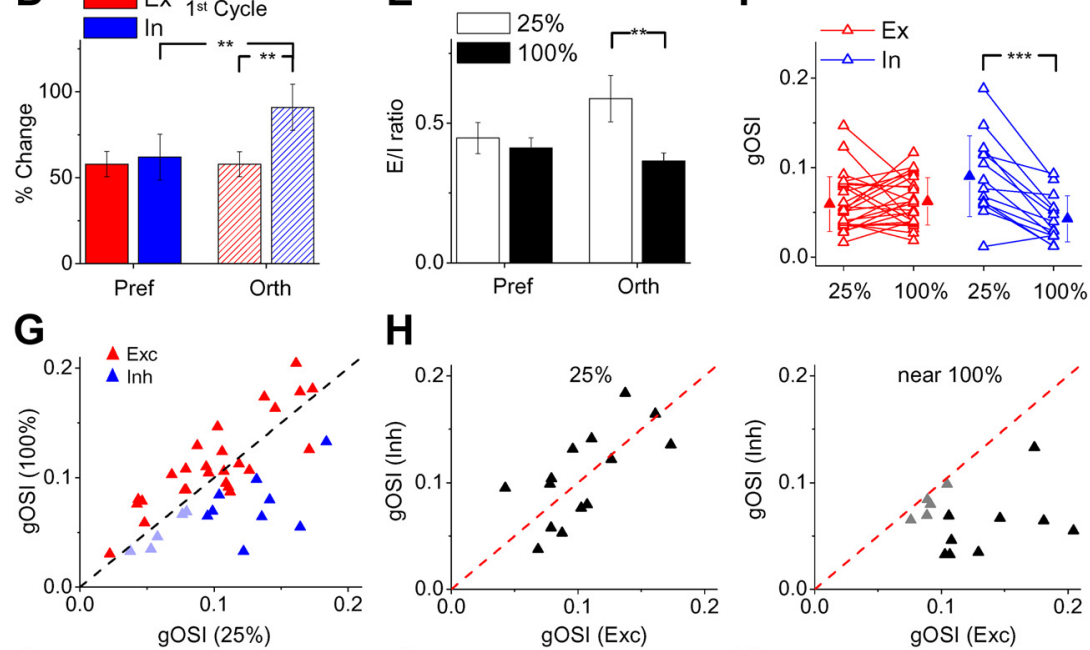

$\mathrm{H}$
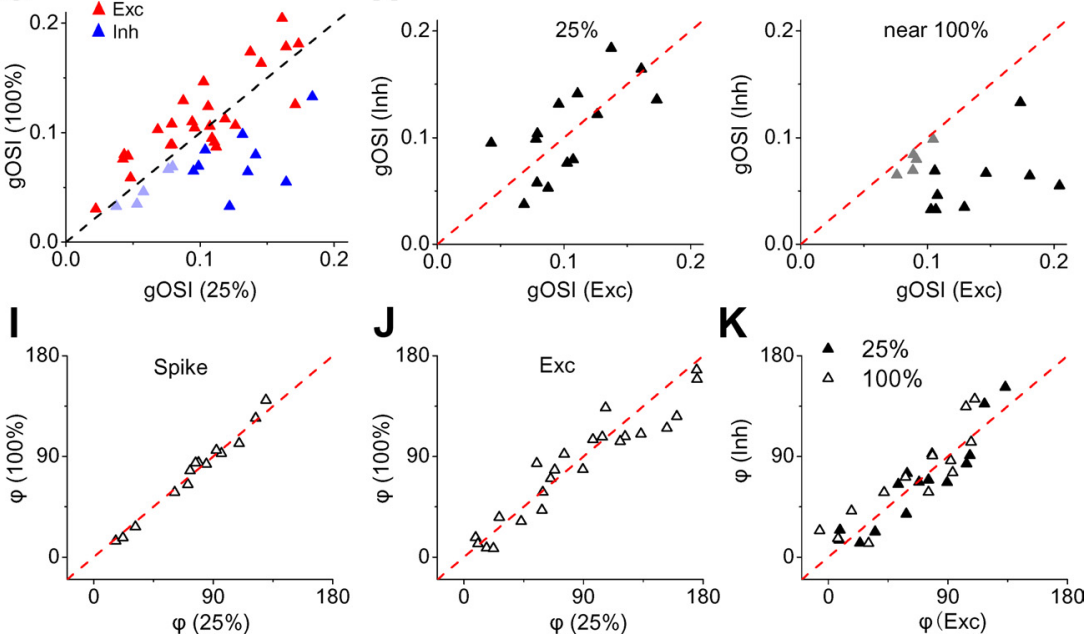

Figure 3. Contrast-dependent changes of synaptic inputs to excitatory neurons. $\boldsymbol{A}$, Percentage change in response level at the preferred and orthogonal orientations, quantified by the peak amplitude of the cycle-averaged response waveform. Bar, SE; $n=$ 14. ${ }^{* *} p<0.01,{ }^{* * *} p<0.001$, paired $t$ test. $\boldsymbol{B}, E / /$ ratio for the two orientations at different contrasts. ${ }^{* *} p<0.01$, paired $t$ test. C, g0SIs of excitation (red, $n=26$ ) and inhibition (blue, $n=14$ ) at $25 \%$ and near $100 \%$ contrast. Data points for the same cell are connected with a line. ${ }^{* *} p<0.001$, paired $t$ test. $\boldsymbol{D}-\boldsymbol{F}$, Summary results based on quantifications of the peak amplitude within the first stimulus cycle. Data are presented in the same way as in A-C. G, Plot of the mean of bootstrapped g0SIs at near $100 \%$ contrast versus that at $25 \%$ contrast for individual cells. For inhibition, dark blue indicates cells that exhibit significant deviation from the identity line $(p<0.01)$. $\boldsymbol{H}$, Plot of the mean of bootstrapped gOSIs for inhibition versus that for excitation to the same cell. Left, At 25\% contrast. Right, At near 100\% contrast. Solid black, Cells that exhibit significant deviation from the identity line ( $p<$ 0.01). I, Preferred orientation of spike response at near $100 \%$ contrast versus that at $25 \%$. J, Preferred orientation of excitation at near $100 \%$ contrast versus that at $25 \%$. $\boldsymbol{K}$, Preferred orientation of inhibition versus that of excitation.

averaged response traces by trials and cycles, and then measured the peak amplitude after smoothing the response trace with a $40 \mathrm{~ms}$ sliding window for averaging. The values of this peak amplitude were used to plot the synaptic tuning curves (Fig. $2 B, D, F, H$ ). In both cells, excitatory and inhibitory responses at all orientations were strengthened with increasing contrast, so that the offsets of the synaptic tuning curves were elevated. While the excitatory response was similarly tuned (Fig. $2 F$ ) or became slightly more sharply tuned (Fig. $2 B$ ) at the high contrast, the inhibitory tuning on the other hand apparently broadened (Fig. 2D,H).

We recorded excitatory responses from 26 neurons. In 14 of these cells, recording was maintained long enough so that inhibitory responses were also completely examined. The summarized results show that from $25 \%$ to near $100 \%$ contrast, excitation and inhibition were increased markedly by $>50 \%$ (Fig. $3 A$ ). The rel- ative increase of excitation was similar between the preferred and orthogonal responses, suggesting a nearly multiplicative change of excitatory input across orientations. The relative increase of inhibition at the preferred orientation was similar to that of excitation, but the relative increase of inhibition at the orthogonal orientation was much greater (Fig. $3 A$ ). Due to these unbalanced increases in excitation and inhibition, the E/I ratio at the orthogonal orientation was significantly decreased (Fig. $3 B$ ), along side a reduction of inhibitory tuning selectivity (Fig. 3C). Meanwhile, excitatory tuning selectivity tended to be slightly enhanced (Fig. 3C). A similar conclusion on a reduced inhibitory tuning selectivity could be made when the peak amplitude during the first cycle was measured (Fig. $3 D, E, F$ ), or when the average conductance was measured (for inhibition, gOSI was $0.11 \pm$ 0.02 at $25 \%$ contrast, $0.05 \pm 0.01$ at near $100 \%$ contrast, mean \pm SE, $p<0.01$, paired $t$ test; for excitation, gOSI was $0.06 \pm 0.03$ at $25 \%$ contrast, $0.08 \pm 0.03$ at near $100 \%$ contrast, $p>0.05)$. Nine of the 14 recorded cells exhibited a significant decrease in inhibitory tuning selectivity (Fig. 3G). As a result, at near $100 \%$ contrast inhibitory tuning selectivity was significantly lower than excitatory tuning selectivity in 9 of 14 cells (Fig. $3 H$, right). Despite the changes in synaptic strength and selectivity, there was no significant change in preferred orientation for either spike or excitatory response (Fig. $3 I, J$ ), nor in similarity between preferred orientations of excitation and inhibition (Fig. $3 K$ ).

\section{An inhibitory synaptic mechanism underlying contrast-dependent sharpening of OS}

Both an increase in excitatory selectivity and a decrease in inhibitory selectivity can lead to an elevation of gOSI of spiking response. Considering the magnitude of the respective change in excitatory and inhibitory tuning (Fig. 3C,F), it can be predicted that the reduced inhibitory tuning selectivity would play a major role. To confirm this point, we simulated contrast-dependent membrane potential responses with a conductance-based integrate-and-fire neuron model, using parameter values (e.g., synaptic strength and synaptic tuning profile) as observed in our experiments. We first averaged the normalized synaptic tuning curves of all the cells. Again, the summary shows that the average excitatory tuning curve barely changed at the high contrast, whereas the average inhibitory tuning curve significantly broadened (Fig. 4A). We simulated waveforms of visually evoked synaptic conductances (in 1 cycle) with a skew normal function (Fig. $4 B$, top), with the inhibitory response delayed relative to the excitatory response by $25 \mathrm{~ms}$ (see Materials and Methods). The membrane potential 
response was then derived by feeding the simulated synaptic conductances into the neuron model (Fig. $4 B$, bottom). To simulate synaptic tuning curves for the model, the average recorded synaptic tuning curves were fitted with Gaussian functions (Fig. 4C). For simplicity, the excitatory tuning curves at the low and high contrasts were chosen to be the same. As shown in Figure $4 D$, the tuning curve of the derived postsynaptic membrane potential response (PSP) was sharper when the synaptic amplitudes and tuning profiles at near $100 \%$ contrast (red) were used in the model, compared with those at $25 \%$ contrast (solid black). The sharper tuning was due to a much larger elevation of PSP response at the preferred than orthogonal orientation (Fig. 4D). This simulation result is largely consistent with our experimental observation that with contrast increases the spiking response was markedly increased at the preferred orientation but remained unchanged at the orthogonal (Fig. 1H). The sharpening of PSP response can be primarily attributed to the increased broadness of inhibitory tuning, because strengthening the excitatory and inhibitory inputs normally while artificially retaining the initial inhibitory tuning profile (as at $25 \%$ contrast) resulted in even weaker tuning of PSP response (Fig. 4D, dashed curve). This result in fact supports the prediction from our previous modeling work that a balanced increase of excitation and inhibition would result in a weakening of tuning selectivity of PSP response and that a broadening of inhibition might be required to counteract this effect (Liu et al., 2011).

To further confirm that a sharpening of PSP response occurs due to the contrastdependent broadening of inhibition, we performed current-clamp recordings from layer 4 excitatory neurons, using a $\mathrm{K}^{+}$gluconate-based internal solution (see Materials and Methods). The subthreshold membrane potential $\left(V_{\mathrm{m}}\right)$ response was obtained after removing spikes with a median filter (Fig. $4 E$, left). Although the $V_{\mathrm{m}}$ response was much more weakly tuned than the spiking response (Fig. $4 E$, right), we did observe that the gOSI of $V_{\mathrm{m}}$ response significantly increased from $25 \%$ to near $100 \%$ contrast (Fig. $4 F$ ). In the meantime, the elevation of $V_{\mathrm{m}}$ response from $25 \%$ to near $100 \%$ contrast was larger at the preferred than at the orthogonal orientation (Fig. 4G, left). Considering that the subthreshold membrane depolarization can be clipped by the spike threshold, the elevation of $V_{\mathrm{m}}$ response at the preferred orientation may even be underestimated. Thus, as expected from the modeling result, the $V_{\mathrm{m}}$ response was indeed sharpened at the high contrast. Furthermore, using the same neuron model, we derived PSP responses from ex-

A
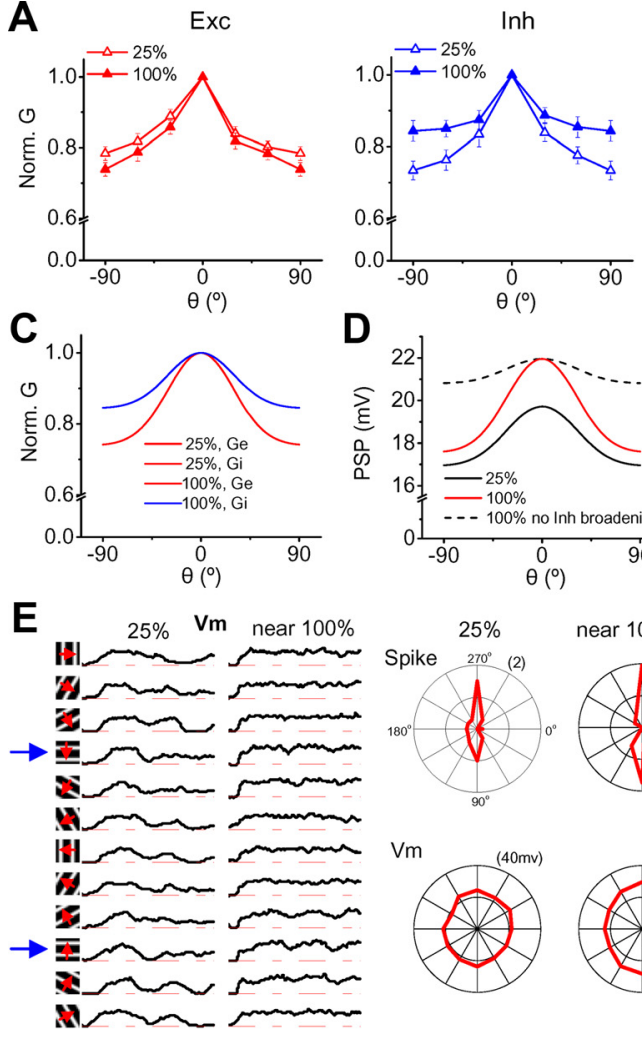

B
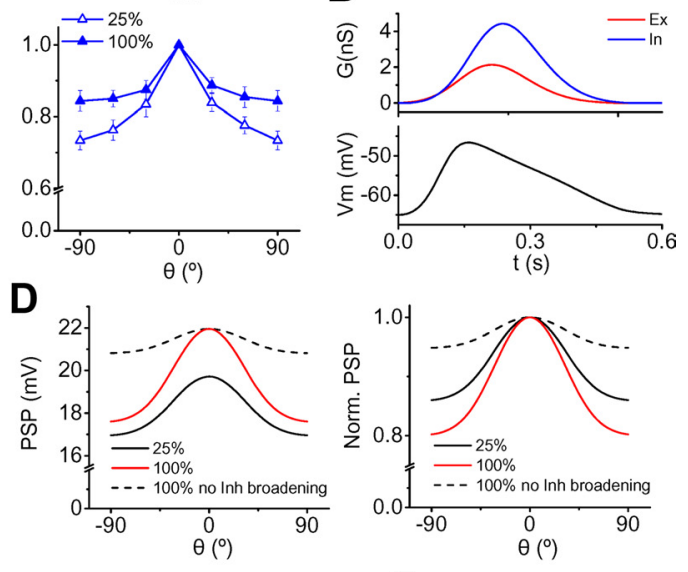

$\mathbf{F}$
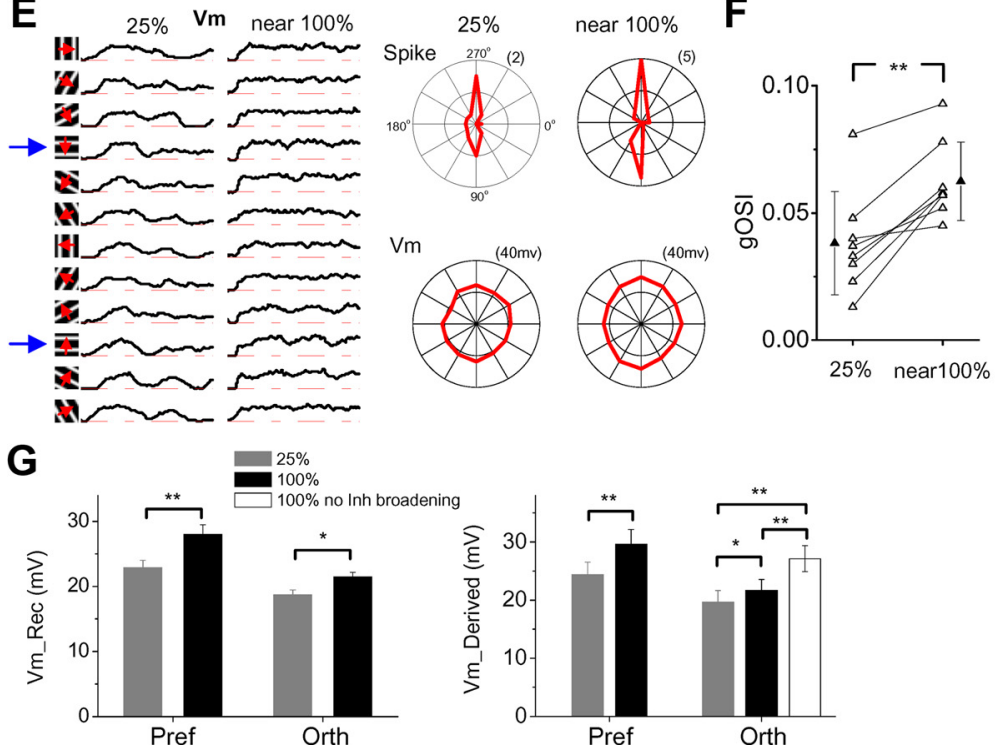

Figure 4. A broadening of inhibitory tuning primarily contributes to the contrast-dependent sharpening of OS. A, Average normalized excitatory and inhibitory tuning curves at the two different contrasts (25\% and near 100\%). Bar, SE; $n=14$. B, Top, Simulated excitatory and inhibitory synaptic conductance waveforms (1 cycle). Bottom, Membrane potential response generated by the integrate-and-fire neuron model when the evoked synaptic conductances shown on top were integrated. $C$, Tuning profiles of excitation and inhibition at 25 and $100 \%$ contrast applied in our model. Ge, excitation. Gi, inhibition. D, Tuning curves of PSP generated by our model. Black, $25 \%$ contrast. Red, 100\% contrast. Dash, 100\% contrast but with the inhibitory tuning profile prevented from broadening (i.e., it is the same as the one at $25 \%$ contrast). The applied strength of synaptic conductance at different contrasts was according to the tuning curve shown in C. Right, Normalized PSP tuning curves. E, Left, Average subthreshold $V_{m}$ responses of an example cell recorded under the current-clamp mode. Spikes were removed by a 15 ms median filter before averaging. Each trace is $1.5 \mathrm{~s}$. Right, Polar graphs of evoke spike rate and peak depolarization voltage. $F$, gOSI of peak $V_{\mathrm{m}}$ response at $25 \%$ and near $100 \%$ contrast. Data points for the same cell are connected with a line. ${ }^{* *} p<0.01$, paired $t$ test; $n=8$. G, Left, Average peak $V_{\mathrm{m}}$ responses at the preferred and orthogonal orientations from our current-clamp recording results. $n=8$. Right, $V_{m}$ responses derived by feeding the experimentally determined excitatory and inhibitory conductances into the neuron model. White column represents the $V_{m}$ response generated when the inhibitory tuning was prevented from broadening (i.e., the recorded inhibitory responses at near $100 \%$ contrast are scaled according to the average inhibitory tuning curve at $25 \%$ contrast). ${ }^{*} p<0.05 ;{ }^{* *} p<0.01$, paired $t$ test. $n=14$.

perimentally determined excitatory and inhibitory conductances (Fig. 4G, right). For the PSP derived in this manner, the response elevation from the low to high contrast at the orthogonal orientation was indeed much smaller than that at the preferred. However, it was markedly increased if we forced the inhibitory tuning not to become broadened by scaling the recorded inhibitory responses at near $100 \%$ contrast according to the average inhibitory tuning curve at $25 \%$ contrast (Fig. 4G, right, white column). Together, our simulation data strongly suggest that the contrast-dependent broadening of inhibition plays a critical role in maintaining sharp orientation tuning at high contrast. 
A

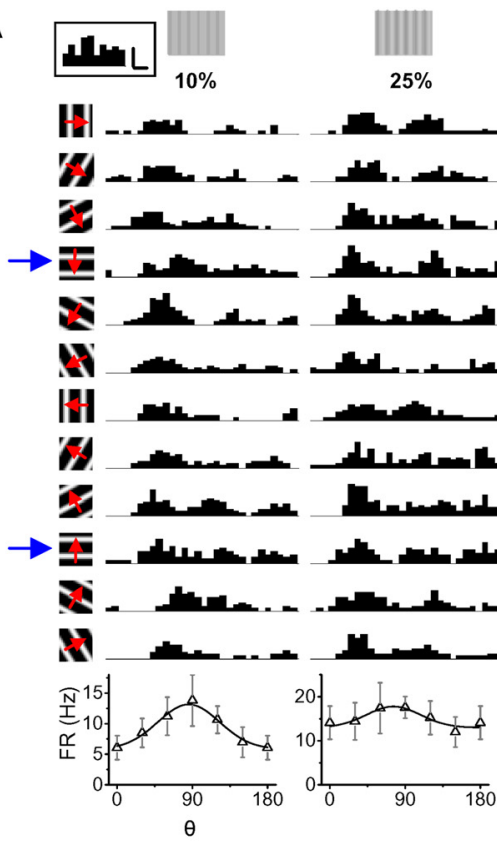

$\underset{50 \%}{||||||} \underset{\text { near } 100 \%}{||||||}$
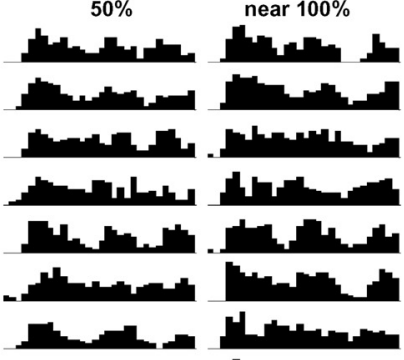

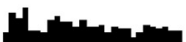
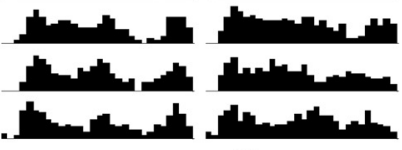

Intm
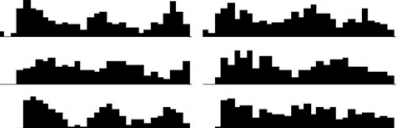

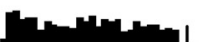

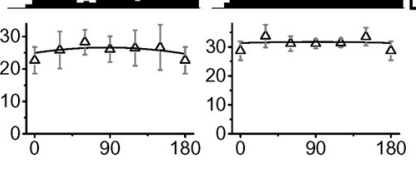

D 0.3
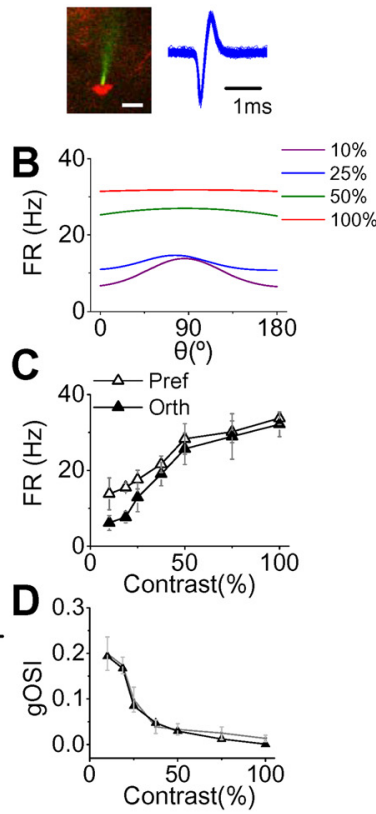

E
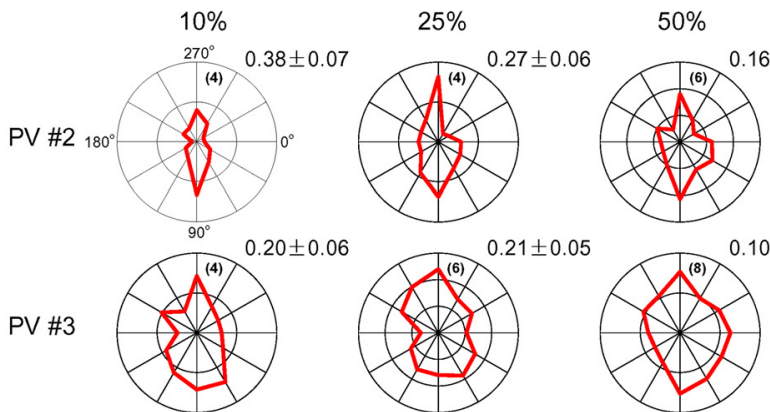

near $100 \%$

$0.04 \pm 0.02$
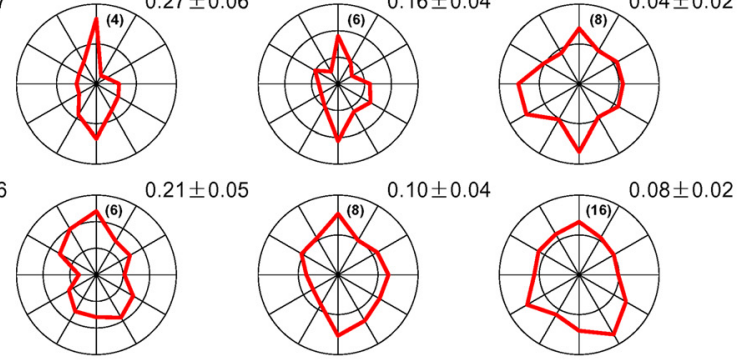

F
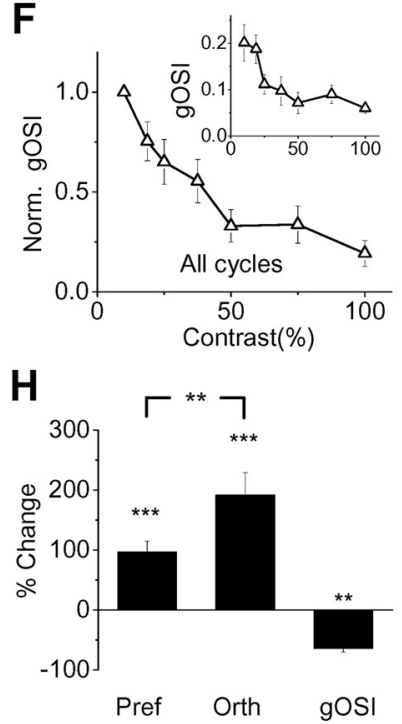

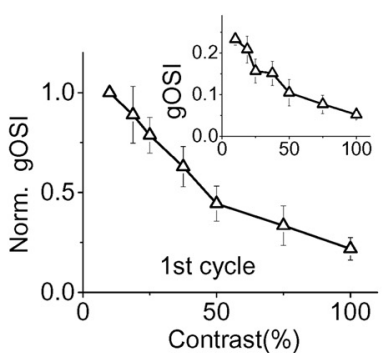

I

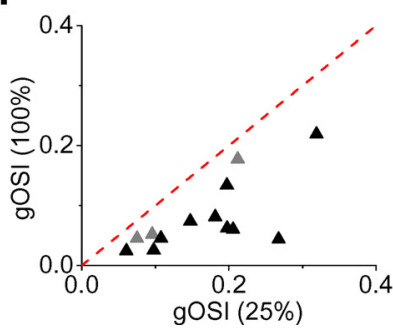

G

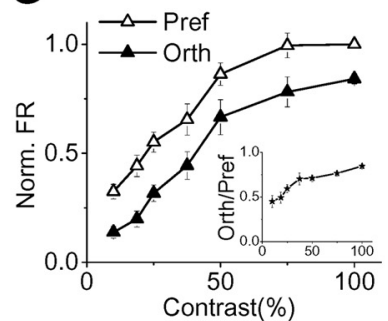

J

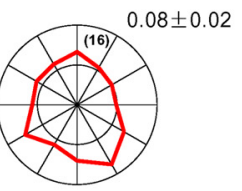

\section{G}

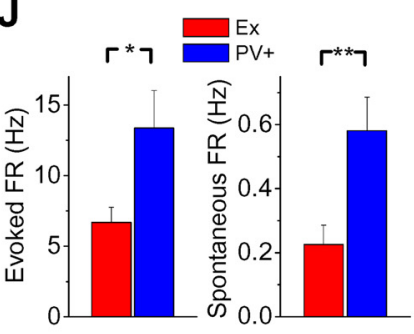

Figure 5. Contrast-dependent broadening of spike response tuning of PV inhibitory neurons. $A$, Poststimulus spike time histograms (PSTHs) for spike responses of an example PV neuron in layer 4. Calibrations: $30 \mathrm{~Hz}, 100 \mathrm{~ms}$. Boxed inset, Top left, PSTH for spontaneous activity. Calibrations: $3 \mathrm{~Hz}, 150 \mathrm{~ms}$. Inset, Top right, Two-photon image of the PV neuron (red) patched by the glass electrode (green), and superimposed 50 individual spike waveforms of the cell. Note that the interval between the trough and the peak is much shorter compared with excitatory neurons. Calibration: $15 \mu \mathrm{m}$. $\boldsymbol{B}$, Superimposed fitted tuning curves at different contrasts. C, Evoked spike rates at the preferred and orthogonal orientations plotted against contrast for the same cell. $D$, gOSI plotted against contrast for the same cell. Gray shows the mean \pm SD of bootstrapped gOSIs (resamplings, 1000 times). $\boldsymbol{E}$, Polar graphs of evoked spike rate for another two PV neurons. $\boldsymbol{F}$, Average gOSI (normalized to the value at the lowest contrast) plotted against contrast. Inset, Average gOSI (absolute value) at different contrasts. Bar, SE; $n=13$. G, Average evoked spike rates (normalized to the value of preferred orientation at near 100\% contrast) at the preferred and orthogonal orientations. Inset, Ratio of spike rate at the orthogonal versus preferred orientation. $\boldsymbol{H}$, Percentage change in evoked spike rate and g0SI value from $25 \%$ to near $100 \%$ contrast. I, Plot of the mean of bootstrapped g0SIs at near $100 \%$ contrast versus that at $25 \%$ contrast for individual cells. Solid black, Cells that exhibit significant deviation from the identity line (bootstrap test, $p<0.01$ ). J, Average evoked (at near $100 \%$ contrast) and spontaneous spike rate for excitatory and PV neurons. ${ }^{*} p<0.05,{ }^{* *} p<0.01, t$ test. 

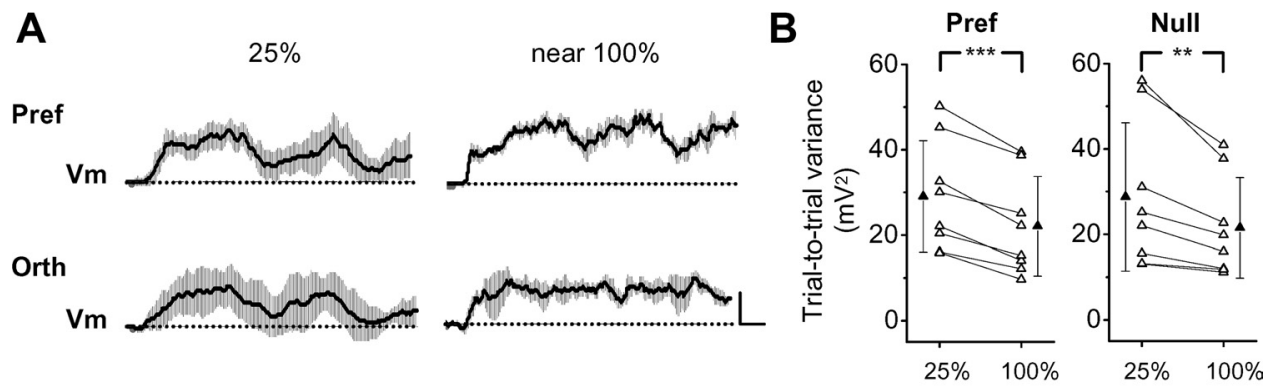

C

D
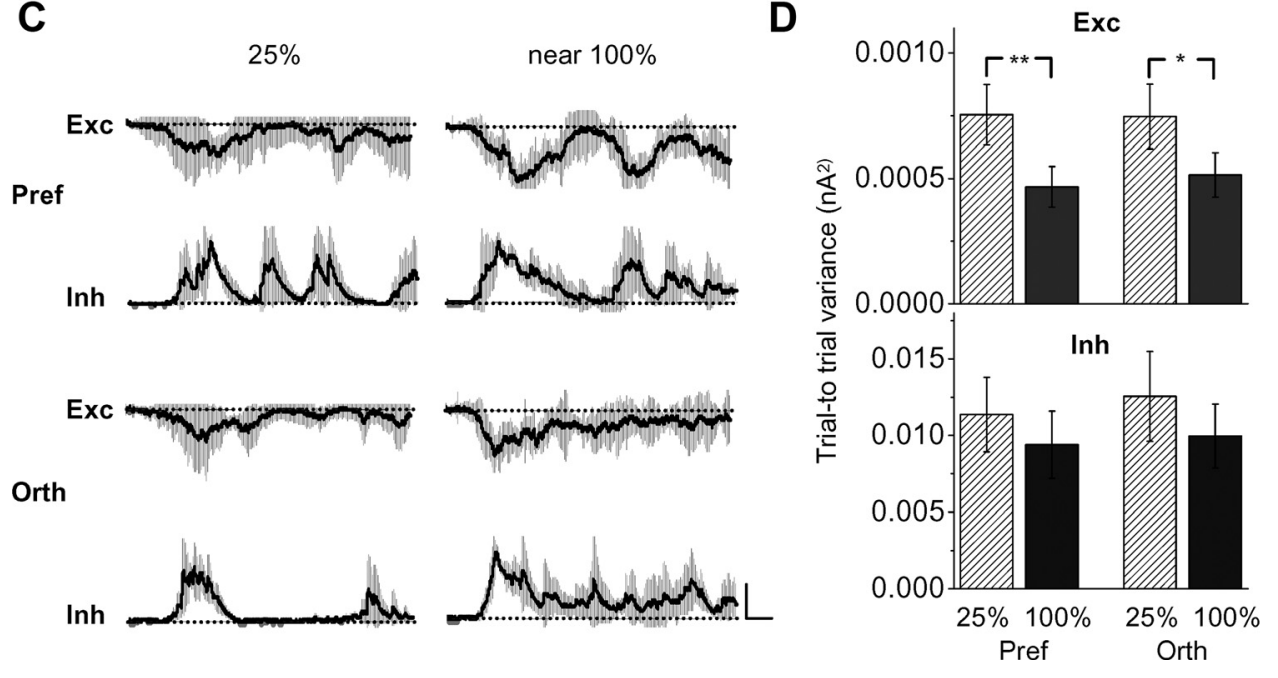

Figure 6. Trial-to-trial variability of synaptic responses. $A$, Average traces of membrane potential responses of an example cell at the preferred (Pref) and orthogonal ( 0 rth) orientations. Shading indicates SD, which was calculated within a $40 \mathrm{~ms}$ sliding window. Red dash line, Baseline. Calibrations: $10 \mathrm{mV}, 200 \mathrm{~ms}$. B, Trial-to-trial variance of membrane potential responses at $25 \%$ and near $100 \%$ contrast for the preferred and orthogonal orientations. $n=8$ cells. ${ }^{* *} p<0.01$; ${ }^{* * *} p<0.001$, paired $t$ test. C, Average traces of excitatory (Exc) and inhibitory (Inh) responses of an example cell at the preferred and orthogonal orientations and at both $25 \%$ and near $100 \%$ contrasts, with SD indicated by the shading. Calibrations: Exc, $30 \mathrm{pA} ;$ Inh, $82 \mathrm{pA}, 200 \mathrm{~ms}$. D, Summary of trial-to-trial variance of synaptic responses. The maximum SD for each orientation was selected for this quantification. $n=14$ cells. ${ }^{* *} p<0.05 ;{ }^{* *} p<0.01$, paired $t$ test.

Contrast-dependent broadening of OS of inhibitory neurons How does the inhibitory tuning become broadened at high contrast? One possibility is that the spiking response tuning of individual inhibitory neurons becomes broadened. PV fast-spiking neurons constitute the largest inhibitory neuron population in layer 4 (Kawaguchi and Kubota, 1997; Gonchar et al., 2007; Xu et al., 2010), yet contrast-dependent tuning properties of these neurons have not been characterized. We thus recorded from PV neurons by two-photon imaging-guided loose-patch recordings in the PV-Cre tdTomato transgenic mice (see Materials and Methods), and in two cases by identifying fast-spiking neurons in blind recordings (Liu et al., 2009). We found that PV neurons exhibited very different behaviors from excitatory neurons. As shown by an example cell in Figure $5 A$, the cell displayed relatively well tuned spiking responses at $10 \%$ contrast. However, the response tuning became more and more flattened with increasing contrast. At near $100 \%$ contrast, its tuning selectivity was almost completely lost (Fig. 5B). Different from excitatory neurons, the response level at both the preferred and orthogonal orientations increased robustly (Fig. 5C), resulting in an almost zero gOSI at the highest contrast (Fig. 5D). A weakening of tuning selectivity was also evident from the polar graphs for another two example PV cells (Fig. 5E). Summarized results for 13 PV neurons show that their tuning selectivity was monotonically decreasing with increasing contrast (Fig. 5F). Their spike responses at the preferred and orthogonal orientations were both elevated, but not in the same rate (Fig. 5G). The relative increase of spike rate was much higher at the orthogonal orientation than at the preferred, resulting in significantly reduced tuning selectivity at near $100 \%$ contrast (Fig. 5H). For individual cells, bootstrap test revealed that 10 of 13 cells exhibited a significant reduction in gOSI at the high contrast (Fig. 5I). Finally, consistent with previous reports (Ma et al., 2010; Atallah et al., 2012), PV neurons had significantly higher spontaneous and evoked firing rates than excitatory neurons (Fig. 5J). Together, our results strongly suggest that PV inhibitory neurons are not contrast invariant. The broadening of orientation tuning of these neurons likely contributes to the broadening of synaptic inhibition to excitatory cells.

\section{Trial-to-trial variability of synaptic responses}

Previous studies in the cat have suggested that contrastdependent changes in trial-to-trial variability is sufficient for generating contrast invariance without reliance on inhibition (Finn et al., 2007; Priebe and Ferster, 2008). Here we looked into this issue and found that the trial-to-trial variability of membrane potential responses was indeed reduced at the high contrast for both the preferred and orthogonal orientations (Fig. 6A,B). At the synaptic level, we found that the variability of excitatory responses significantly decreased at the high contrast (Fig. 6C,D), consistent with the notion of a reduced variability of thalamic inputs (Sadagopan and Ferster, 2012). The variability of inhibitory responses also tended to decline, although the level of reduction did not reach statistical significance (Fig. $6 C, D$ ). Thus, the change in variability of excitatory responses directly contributes to that of membrane potential responses. In addition to broadened inhibition, the reduced response variability can also con- 

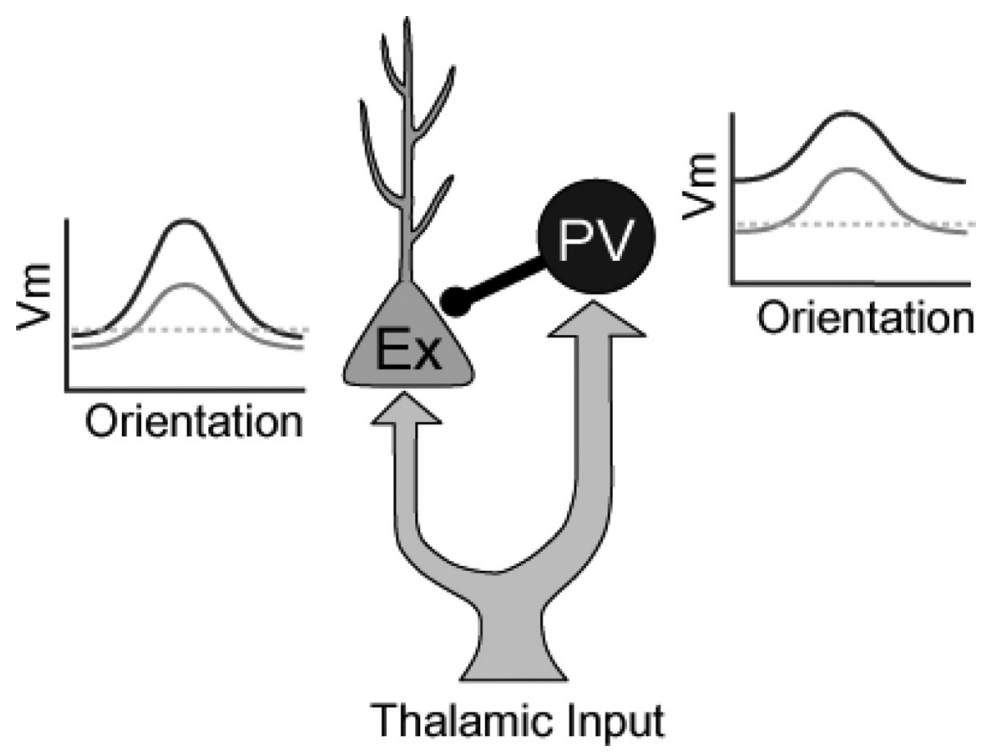

Figure 7. A simple model for contrast invariance of excitatory neurons and contrast variance of PV inhibitory neurons. The schematic orientation tuning curves of $V_{m}$ response for the PV neuron(s) and the excitatory (Ex) cell are shown. Gray, At a low contrast. Black, At a high contrast. Dotted gray line, Spike threshold. The PV neuron(s) exhibits a similar preferred orientation as the excitatory cell, since they receive a similar set of thalamic inputs.

tribute to preventing elevations of the null response, since it is equivalent to elevating the spike threshold (Finn et al., 2007; Priebe and Ferster, 2008).

\section{Discussion}

\section{Contrast invariance versus contrast-dependent sharpening} of OS

Contrast invariance is traditionally defined as a constant orientation tuning width in the face of contrast increases. Meanwhile, it has been reported for ferret and a subset of primate visual cortical neurons that a global measure of OS (e.g., circular variance) exhibits contrastdependent changes, which indicate an increased selectivity level at high contrast (Alitto and Usrey, 2004; Johnson et al., 2008). Our data on excitatory neuron tuning measured with gOSI are consistent with the results in these species in that tuning selectivity is enhanced with increasing contrast. The data on tuning width are also consistent with a previous report in the mouse V1 showing that tuning width is not significantly changed from midcontrast to high contrast (50-100\%) (Niell and Stryker, 2008). For a broader range of contrasts, our data show that orientation tuning width is in fact reduced with increasing contrast. This general trend of decreasing tuning width, however, does not contradict the notion of contrast invariance at a mechanistic level. Tuning width being unchanged or reduced, the task the cortical circuits need to achieve is the same: to keep selectivity sharp at high contrast. The details of contrast-dependent changes of orientation tuning may depend on different species, cortical layers, or cell types studied. Our results showing that the spike rate of excitatory neurons at the orthogonal orientation is not enhanced with increasing contrast highlights the essence of the problem the visual cortex is commonly facing: how to prevent an elevation of the null response while there is certainly a large increase of excitatory drive at the null orientation.

\section{Inhibitory contribution to contrast-dependent sharpening of OS}

In our previous study of OS in layer $2 / 3$ simple cells, we found that inhibitory input is more broadly tuned than excitatory input (Liu et al., 2011; Atallah et al., 2012). Such broader inhibition can significantly sharpen the tuning of output response compared with cotuned inhibition (Liu et al., 2011). We also found in our initial modeling work that simply scaling up excitation and inhibition in proportion would not lead to invariant tuning but a broadening of output response (Liu et al., 2011). We have thus hypothesized that differential contrast-dependent modulations of excitatory and inhibitory tuning might be necessary for achieving contrast invariance.

In the current study, we have indeed observed that contrast changes modulate the two types of synaptic input in different manners. Excitatory responses evoked by different orientations are strengthened nearly multiplicatively, consistent with contrast-dependent increases in firing rate of thalamic neurons (Finn et al., 2007; Olsen et al., 2012). Inhibitory responses are also strengthened, but with a relatively greater level at the orthogonal orientation than at the preferred, resulting in a broadening of the inhibitory tuning. This can be attributed to a contrast-dependent broadening of spiking response tuning of individual inhibitory neurons, and additionally a potential increase in convergence of inhibitory neuron inputs, which remains to be investigated. Our modeling results indicate that the broadening of inhibition at high contrast largely limits the elevation of membrane potential response at the null orientation despite the greatly increased excitatory conductance. Otherwise, if inhibition is not broadened, the elevation of the null $V_{\mathrm{m}}$ response would be similar to (or even greater than) that of the preferred response, leading to a reduced selectivity of output response (Fig. 4D, dash curve; Fig. 4G, white column). Thus, our data indicate that an inhibitory mechanism plays a critical role in preventing a significant elevation of the null response at high contrast.

\section{Trial-to-trial variability of synaptic responses}

Previous studies in the cat have shown that the trial-to-trial variability of membrane potential responses of simple cells is reduced with increasing contrast (Anderson et al., 2000). It can be attributed to a reduced variance of thalamic inputs, and is sufficient for accounting for the contrast invariance of simple cells (Finn et al., 2007; Priebe and Ferster, 2008; Sadagopan and Ferster, 2012). In the current study, we also observed a reduction of trial-to-trial variability of membrane potential responses, which can be directly attributed to a decrease in variability of excitatory responses. In addition, due to a saturating input-output transfer function as discussed in our previous study (Liu et al., 2011), an increase in excitatory conductance with a fixed variance would naturally result in a reduced variance of membrane potential response. Thus, two factors (increased excitatory conductance and increased variance of excitatory conductance) together can account for the reduced variability of membrane potential responses at high contrast. The observed changes in membrane potential response variability, however, cannot sufficiently explain the contrast-dependent changes in spike-response tuning in the current study. This is because, in the absence of inhibitory broadening, there would be a large increase in the null $V_{\mathrm{m}}$ re- 
sponse (Fig. $4 D, G$ ). Reducing variability, which is equivalent to elevating the effective spike threshold, would be incapable of suppressing the null spike response while allowing a large increase in the preferred spike response, since it affects both responses at the same time. After the broadened inhibition largely limits the elevation of the null $V_{\mathrm{m}}$ response, the reduction in variability can further contribute to suppressing the null spike response by elevating the spike threshold. This may explain why there is an increase in the null $V_{\mathrm{m}}$ response at high contrast (Fig. $4 G$ ), whereas the null spike response is not changed (Fig. 1H). It should be noted that the level of inhibitory contribution to orientation selectivity may differ in different species.

\section{Contrast-dependent broadening of orientation tuning of inhibitory neurons}

An interesting observation in this study is that orientation tuning of PV inhibitory neurons becomes flattened as contrast increases. The largely untuned property of PV neurons at high contrast is consistent with observations from several previous studies that examined PV neurons at a fixed contrast (Kerlin et al., 2010; Ma et al., 2010; Hofer et al., 2011; Kuhlman et al., 2011; Zariwala et al., 2011; Atallah et al., 2012). In addition, the contrastdependent changes of PV neuron tuning may provide an explanation for the discrepancy of PV neuron selectivity reported by different groups (e.g., Kerlin et al., 2010; Runyan et al., 2010), since different absolute contrasts may have been applied. Interestingly, the behavior of PV neurons in response to contrast increases (i.e., concurrent increases in firing rate at all orientations) is exactly what has been predicted for excitatory neurons by the original Hubel and Wiesel model, which did not take inhibition or variability into account (Ferster and Miller, 2000). In layer 4, PV neurons have been shown to receive thalamic input like excitatory neurons and to provide them with fast feedforward inhibition (Gibson et al., 1999; Gabernet et al., 2005; Cruikshank et al., 2007). Here, we propose that a simple thalamocortical circuit recruiting feedforward inhibition can explain both the contrast-dependent sharpening of excitatory neurons and the contrast-dependent broadening of PV inhibitory neurons (Fig. 7). In this circuit, PV neurons provide primary feedforward inhibition to the excitatory neuron of interest. The PV neurons and the excitatory neuron receive similar thalamic inputs, as evidenced by a similar preferred orientation between excitatory and inhibitory tuning across contrasts (Fig. $3 K$ ). With contrast increases, the thalamic inputs are strengthened, increasing excitatory drive onto both the PV neurons and the excitatory cell. As PV neurons receive relatively stronger thalamic input compared with excitatory cells (Gabernet et al., 2005; Cruikshank et al., 2010; Schiff and Reyes, 2012), the increase in excitatory drive onto PV neurons can easily lead to an elevation of their firing rate at all orientations due to the "iceberg" effect (Carandini and Ferster, 2000; Wehr and Zador, 2003; Zhang et al., 2003). The strengthened and broadened inhibitory input from PV neurons can then counteract the increased excitatory drive onto the excitatory cell, and maintain or even enhance the sharpness of orientation tuning of its output response. Such synaptic circuitry mechanism may also function in other sensory systems to achieve sharp tuning regardless of stimulus intensity.

\section{References}

Albrecht DG, Hamilton DB (1982) Striate cortex of monkey and cat: contrast response function. J Neurophysiol 48:217-237.

Alitto HJ, Usrey WM (2004) Influence of contrast on orientation and temporal frequency tuning in ferret primary visual cortex. J Neurophysiol 91:2797-2808.
Anderson JS, Lampl I, Gillespie DC, Ferster D (2000) The contribution of noise to contrast invariance of orientation tuning in cat visual cortex. Science 290:1968-1972.

Atallah BV, Bruns W, Carandini M, Scanziani M (2012) Parvalbuminexpressing interneurons linearly transform cortical responses to visual stimuli. Neuron 73:159-170.

Borg-Graham LJ, Monier C, Frégnac Y (1998) Visual input evokes transient and strong shunting inhibition in visual cortical neurons. Nature 393:369-373.

Carandini M, Ferster D (2000) Membrane potential and firing rate in cat primary visual cortex. J Neurosci 20:470-484.

Carandini M, Heeger DJ, Movshon JA (1997) Linearity and normalization in simple cells of the macaque primary visual cortex. J Neurosci 17:8621-8644.

Celebrini S, Thorpe S, Trotter Y, Imbert M (1993) Dynamics of orientation coding in area V1 of the awake primate. Vis Neurosci 10:811-825.

Cruikshank SJ, Lewis TJ, Connors BW (2007) Synaptic basis for intense thalamocortical activation of feedforward inhibitory cells in neocortex. Nat Neurosci 10:462-468.

Cruikshank SJ, Urabe H, Nurmikko AV, Connors BW (2010) Pathwayspecific feedforward circuits between thalamus and neocortex revealed by selective optical stimulation of axons. Neuron 65:230-245.

De Valois RL, Yund EW, Hepler N (1982) The orientation and direction selectivity of cells in macaque visual cortex. Vision Res 22:531-544.

Efron B, Tibshirani R (1993) An introduction to the bootstrap. London: Chapman and Hall.

Ferster D, Miller KD (2000) Neural mechanisms of orientation selectivity in the visual cortex. Annu Rev Neurosci 23:441-471.

Finn IM, Priebe NJ, Ferster D (2007) The emergence of contrast-invariant orientation tuning in simple cells of cat visual cortex. Neuron 54:137-152.

Gabernet L, Jadhav SP, Feldman DE, Carandini M, Scanziani M (2005) Somatosensory integration controlled by dynamic thalamocortical feedforward inhibition. Neuron 48:315-327.

Gibson JR, Beierlein M, Connors BW (1999) Two networks of electrically coupled inhibitory neurons in neocortex. Nature 402:75-79.

Gonchar Y, Wang Q, Burkhalter A (2007) Multiple distinct subtypes of GABAergic neurons in mouse visual cortex identified by triple immunostaining. Front Neuroanat 1:3.

Hofer SB, Ko H, Pichler B, Vogelstein J, Ros H, Zeng H, Lein E, Lesica NA, Mrsic-Flogel TD (2011) Differential connectivity and response dynamics of excitatory and inhibitory neurons in visual cortex. Nat Neurosci 14:1045-1052.

Hubel DH, Wiesel TN (1962) Receptive fields, binocular interaction and functional architecture in the cat's visual cortex. J Physiol 160:106-154.

Johnson EN, Hawken MJ, Shapley R (2008) The orientation selectivity of color-responsive neurons in macaque V1. J Neurosci 28:8096-8106.

Kawaguchi Y, Kubota Y (1997) GABAergic cell subtypes and their synaptic connections in rat frontal cortex. Cereb Cortex 7:476-486.

Kerlin AM, Andermann ML, Berezovskii VK, Reid RC (2010) Broadly tuned response properties of diverse inhibitory neuron subtypes in mouse visual cortex. Neuron 67:858-871.

Kuhlman SJ, Tring E, Trachtenberg JT (2011) Fast-spiking interneurons have an initial orientation bias that is lost with vision. Nat Neurosci 14: $1121-1123$.

Lauritzen TZ, Miller KD (2003) Different roles for simple-cell and complexcell inhibition in V1. J Neurosci 23:10201-10213.

Li CY, Creutzfeldt O (1984) The representation of contrast and other stimulus parameters by single neurons in area 17 of the cat. Pflugers Arch 401:304-314.

Li YT, Ma WP, Pan CJ, Zhang LI, Tao HW (2012) Broadening of cortical inhibition mediates developmental sharpening of orientation selectivity. J Neurosci 32:3981-3991.

Liu BH, Wu GK, Arbuckle R, Tao HW, Zhang LI (2007) Defining cortical frequency tuning with recurrent excitatory circuitry. Nat Neurosci 10: $1594-1600$

Liu BH, Li P, Li YT, Sun YJ, Yanagawa Y, Obata K, Zhang LI, Tao HW (2009) Visual receptive field structure of cortical inhibitory neurons revealed by two-photon imaging guided recording. J Neurosci 29:10520-10532.

Liu BH, Li P, Sun YJ, Li YT, Zhang LI, Tao HW (2010) Intervening inhibition underlies simple-cell receptive field structure in visual cortex. Nat Neurosci 13:89-96.

Liu BH, Li YT, Ma WP, Pan CJ, Zhang LI, Tao HW (2011) Broad inhibition 
sharpens orientation selectivity by expanding input dynamic range in mouse simple cells. Neuron 71:542-554.

Ma WP, Liu BH, Li YT, Huang ZJ, Zhang LI, Tao HW (2010) Visual representations by cortical somatostatin inhibitory neurons-selective but with weak and delayed responses. J Neurosci 30:14371-14379.

Madisen L, Zwingman TA, Sunkin SM, Oh SW, Zariwala HA, Gu H, Ng LL, Palmiter RD, Hawrylycz MJ, Jones AR, Lein ES, Zeng H (2010) A robust and high-throughput Cre reporting and characterization system for the whole mouse brain. Nat Neurosci 13:133-140.

Mangini NJ, Pearlman AL (1980) Laminar distribution of receptive field properties in the primary visual cortex of the mouse. J Comp Neurol 193:203-222.

Mariño J, Schummers J, Lyon DC, Schwabe L, Beck O, Wiesing P, Obermayer K, Sur M (2005) Invariant computations in local cortical networks with balanced excitation and inhibition. Nat Neurosci 8:194-201.

Monier C, Chavane F, Baudot P, Graham LJ, Frégnac Y (2003) Orientation and direction selectivity of synaptic inputs in visual cortical neurons: a diversity of combinations produces spike tuning. Neuron 37:663-680.

Movshon JA, Thompson ID, Tolhurst DJ (1978) Spatial and temporal contrast sensitivity of neurones in areas 17 and 18 of the cat's visual cortex. J Physiol 283:101-120.

Niell CM, Stryker MP (2008) Highly selective receptive fields in mouse visual cortex. J Neurosci 28:7520-7536.

Olsen SR, Bortone DS, Adesnik H, Scanziani M (2012) Gain control by layer six in cortical circuits of vision. Nature 483:47-52.

Priebe NJ, Ferster D (2008) Inhibition, spike threshold, and stimulus selectivity in primary visual cortex. Neuron 57:482-497.

Ringach DL, Hawken MJ, Shapley R (2003) Dynamics of orientation tuning in macaque V1: the role of global and tuned suppression. J Neurophysiol 90:342-352.

Runyan CA, Schummers J, Van Wart A, Kuhlman SJ, Wilson NR, Huang ZJ, Sur M (2010) Response features of parvalbumin-expressing interneurons suggest precise roles for subtypes of inhibition in visual cortex. Neuron 67:847-857.

Sadagopan S, Ferster D (2012) Feedforward origins of response variability underlying contrast invariant orientation tuning in cat visual cortex. Neuron 74:911-923.

Schiff ML, Reyes AD (2012) Characterization of thalamocortical responses of regular-spiking and fast-spiking neurons of the mouse auditory cortex in vitro and in silico. J Neurophysiol 107:1476-1488.
Sclar G, Freeman RD (1982) Orientation selectivity in the cat's striate cortex is invariant with stimulus contrast. Exp Brain Res 46:457-461.

Skottun BC, Bradley A, Sclar G, Ohzawa I, Freeman RD (1987) The effects of contrast on visual orientation and spatial frequency discrimination: a comparison of single cells and behavior. J Neurophysiol 57:773-786.

Somers DC, Nelson SB, Sur M (1995) An emergent model of orientation selectivity in cat visual cortical simple cells. J Neurosci 15:5448-5465.

Tan AY, Zhang LI, Merzenich MM, Schreiner CE (2004) Tone-evoked excitatory and inhibitory synaptic conductances of primary auditory cortex neurons. J Neurophysiol 92:630-643.

Tan AY, Brown BD, Scholl B, Mohanty D, Priebe NJ (2011) Orientation selectivity of synaptic input to neurons in mouse and cat primary visual cortex. J Neurosci 31:12339-12350.

Taniguchi H, He M, Wu P, Kim S, Paik R, Sugino K, Kvitsani D, Fu Y, Lu J, Lin Y, Miyoshi G, Shima Y, Fishell G, Nelson SB, Huang ZJ (2011) A resource of Cre driver lines for genetic targeting of GABAergic neurons in cerebral cortex. Neuron 71:995-1013.

Troyer TW, Krukowski AE, Priebe NJ, Miller KD (1998) Contrast-invariant orientation tuning in cat visual cortex: thalamocortical input tuning and correlation-based intracortical connectivity. J Neurosci 18:5908-5927.

Wehr M, Zador AM (2003) Balanced inhibition underlies tuning and sharpens spike timing in auditory cortex. Nature 426:442-446.

Wu GK, Arbuckle R, Liu BH, Tao HW, Zhang LI (2008) Lateral sharpening of cortical frequency tuning by approximately balanced inhibition. Neuron 58:132-143.

Xing D, Ringach DL, Hawken MJ, Shapley RM (2011) Untuned suppression makes a major contribution to the enhancement of orientation selectivity in macaque V1. J Neurosci 31:15972-15982.

Xu X, Roby KD, Callaway EM (2010) Immunochemical characterization of inhibitory mouse cortical neurons: three chemically distinct classes of inhibitory cells. J Comp Neurol 518:389-404.

Zariwala HA, Madisen L, Ahrens KF, Bernard A, Lein ES, Jones AR, Zeng H (2011) Visual tuning properties of genetically identified layer $2 / 3$ neuronal types in the primary visual cortex of cre-transgenic mice. Front Syst Neurosci 4:162.

Zhang LI, Tan AY, Schreiner CE, Merzenich MM (2003) Topography and synaptic shaping of direction selectivity in primary auditory cortex. Nature 424:201-205.

Zhang M, Liu Y, Wang SZ, Zhong W, Liu BH, Tao HW (2011) Functional elimination of excitatory feedforward inputs underlies developmental refinement of visual receptive fields in zebrafish. J Neurosci 31:5460-5469. 